\title{
QUEEN'S
UNIVERSITY
BELFAST
}

\section{Semi-supervised identification of cancer subgroups using survival outcomes and overlapping grouping information}

Wei, W., Sun, Z., da Silveira, W. A., Yu, Z., Lawson, A., Hardiman, G., Kelemen, L. E., \& Chung, D. (2018). Semi-supervised identification of cancer subgroups using survival outcomes and overlapping grouping information. Statistical Methods in Medical Research, 1-13. https://doi.org/10.1177/0962280217752980

Published in:

Statistical Methods in Medical Research

Document Version:

Peer reviewed version

Queen's University Belfast - Research Portal:

Link to publication record in Queen's University Belfast Research Portal

Publisher rights

Copyright 2019 SAGE. This work is made available online in accordance with the publisher's policies. Please refer to any applicable terms of use of the publisher.

\section{General rights}

Copyright for the publications made accessible via the Queen's University Belfast Research Portal is retained by the author(s) and / or other copyright owners and it is a condition of accessing these publications that users recognise and abide by the legal requirements associated with these rights.

Take down policy

The Research Portal is Queen's institutional repository that provides access to Queen's research output. Every effort has been made to ensure that content in the Research Portal does not infringe any person's rights, or applicable UK laws. If you discover content in the Research Portal that you believe breaches copyright or violates any law, please contact openaccess@qub.ac.uk. 


\section{Semi-supervised Identification of Cancer Subgroups using Survival Outcomes and Overlapping Grouping Information}

Wei Wei ${ }^{1,2}$, Zequn Sun ${ }^{1}$, Willian A. da Silveira ${ }^{3,4}$, Zhenning Yu ${ }^{1}$, Andrew Lawson ${ }^{1}$,

Gary Hardiman ${ }^{1,4,5}$, Linda E. Kelemen ${ }^{1}$, Dongjun Chung ${ }^{1}$

1 Department of Public Health Sciences, Medical University of South Carolina, Charleston,

SC, USA

2 Department of Biostatistics, Yale University, New Haven, CT, USA

3 Department of Pathology and Laboratory Medicine, Medical University of South

Carolina, Charleston, SC, USA

4 Center for Genomic Medicine, Medical University of South Carolina, Charleston, SC, USA

5 Department of Medicine, Medical University of South Carolina, Charleston, SC, USA 


\section{Keywords}

Clustering; variable selection; biological pathway; integrative analysis; cancer genomics;

gene set

\section{Corresponding author}

Dongjun Chung, Department of Public Health Sciences, Medical University of South

Carolina

e-mail: chungd@musc.edu 


\begin{abstract}
Identification of cancer patient subgroups using high throughput genomic data is of critical importance to clinicians and scientists because it can offer opportunities for more personalized treatment and overlapping treatments of cancers. In spite of tremendous efforts, this problem still remains challenging because of low reproducibility and instability of identified cancer subgroups and molecular features. In order to address this challenge, we developed InGRiD (Integrative Genomics Robust iDentification of cancer subgroups), a statistical approach that integrates information from biological pathway databases with high-throughput genomic data to improve the robustness for identification and interpretation of molecularly-defined subgroups of cancer patients. We applied InGRiD to the gene expression data of high-grade serous ovarian cancer from The Cancer Genome Atlas and the Australian Ovarian Cancer Study. The results indicate clear benefits of the pathway-level approaches over the gene-level approaches. In addition, using the proposed InGRiD framework, we also investigate and address the issue of gene sharing among pathways, which often occurs in practice, to further facilitate biological interpretation of key molecular features associated with cancer progression. The $\mathrm{R}$ package 'INGRID' implementing the proposed approach is currently available in our research group GitHub webpage (https://dongjunchung.github.io/INGRID/).
\end{abstract}




\section{Introduction}

During the last decade, The Cancer Genome Atlas (TCGA) Consortium, supported by the U.S. National Cancer Institute (NCI) and the U.S. National Human Genome Research Institute (NHGRI), contributed to significant advances in cancer genomics research to improve our understanding of the molecular basis of cancer subgroups and their associations with prognosis ${ }^{1-6}$. TCGA employs an integrative approach based on multiple genomic platforms, including somatic mutation, copy number alteration (CNA), DNA methylation, and gene expression, to identify shared molecular alterations between subsets of tumors. While this integrative approach provides unprecedented opportunities to investigate a large number of cancer patients from diverse molecular perspectives, the high dimensionality, complexity, and heterogeneity of the data pose challenges for the effective and robust analysis of these datasets. For example, each genomic platform profiles thousands to several ten thousands of genes and generates various data types, including continuous (gene expression and DNA methylation), categorical (CNA), and binary (somatic mutation) measurements. Moreover, driver molecular features are often specific to only certain cancer subgroups and data types ${ }^{7-11}$.

The development of iCluster+ for the analysis of such cancer genomics datasets overcame many of these challenges by simultaneously identifying cancer subgroups and 
important genes from multiple genomic platforms within a unified framework ${ }^{7}$. In spite of this, the following challenges still remain to be solved. First, the identification of key genes does not directly promote understanding of biological networks and additional downstream analyses are needed to characterize these genes in the context of biological networks. Second, gene-level identification has often been reported to not be well reproduced between different studies ${ }^{12}$. Third, genes with weak effect sizes might not be identified in gene-level analyses ${ }^{8}$. These limitations suggest a need for a statistical framework that improves the robustness in biological findings and the interpretation of molecularlydefined subgroups of cancer patients.

One approach to overcome these challenges is to investigate molecular characteristics of cancers at the pathway level, a strategy that has been reported to be more robust and reproducible across studies ${ }^{12,13}$. Summarizing molecular measurements at the pathway level potentially can also lead to improved statistical power because the dimension of molecular data is vastly reduced and moderate signals from multiple genes can be aggregated ${ }^{8,14,15}$. The developers of PARADIGM ${ }^{9}$ made important progress in this direction by summarizing molecular activities in various genomic platforms as pathway-level activation scores. However, in the PARADIGM framework, advantages of pathway-level analyses were still not fully investigated in the context of cancer subgroup 
identification ${ }^{16}$. The pathway index model tried to utilize the pathway information for the purpose of cancer subgroup identification ${ }^{17}$. Specifically, this approach characterizes the prognostic risk of a pathway in two steps. In the first step (gene selection), genes are selected separately for each pathway by LASSO Cox regression and determined as either "cancer susceptible" or "cancer resistant" based on the signs of their LASSO coefficients. In the second step (pathway selection), a pathway index is calculated by comparing the mean expressions of cancer susceptible genes and cancer resistant genes. The number of pathways with positive indices can then be used to classify patients into low, moderate and high risk groups. However, the pathway index model can only tell if a pathway is "in" or "out" and it is not capable of determining the joint effects of pathways or the relative importance of each selected pathway.

In addition to these limitations in pathway-level approaches for cancer subtype identification, the pathway information itself further poses its own challenges. Specifically, despite various attempts to investigate molecular features at the pathway level, the issue of gene overlap between pathways is often ignored. For example, in the widely-used KEGG pathway database ${ }^{18}, 42 \%$ of genes in non-metabolic pathways are members of multiple pathways. Such pathway overlap generates inter-correlation among pathways, which can lead to reduced stability in pathway selection and parameter estimation. Moreover, if 
common genes are strongly associated with an outcome of interest, multiple pathways containing these genes can be artificially identified as important pathways. In this case, the results obtained from pathway-level analyses are difficult to interpret and it is not clear which pathway is truly involved in disease genesis and progression. One approach to deal with this issue is to divide pathways into unique gene sets and their intersections ${ }^{19}$ but this simple approach cannot be applied to more than two pathways. Another approach is to focus on unique genes by down-weighting genes belonging to multiple pathways ${ }^{20}$. However, this approach is biologically questionable because common genes might represent upstream regulators or important cross talks among different biological processes.

In order to address these challenges, we propose InGRiD (Integrative Genomics Robust iDentification of cancer subgroups), a statistical approach that improves the identification of molecularly-defined subgroups of cancer patients in the following aspects. First, we address the pathway overlap issue by redefining the gene set membership of common genes. This approach creates unique membership for each gene, thereby improving the stability and interpretability of the pathway analysis results. Second, we simultaneously represent the gene expression profiles of pathways and select key genes from each pathway by constructing pathway-level latent components using sparse partial least squares (SPLS) Cox regression. Finally, InGRiD allows simultaneous inference in 
multiple biological layers (pathways and genes) within a unified statistical framework without any additional laborious downstream analysis.

\section{Data Description}

We illustrate the usefulness of the proposed InGRiD approach using a cohort of high-grade serous ovarian cancer (HGSOC) patients from The Cancer Genome Atlas (TCGA) project

${ }^{1}$. This cohort consists of 485 patients from whom survival times from diagnosis and gene expression measurements were obtained. The details on collecting and processing TCGA ovarian cancer data can be found elsewhere ${ }^{1}$. None of the TCGA patients had grade 1 disease. The Australian Ovarian Cancer Study (AOCS) ${ }^{21}$ was used as an independent dataset to evaluate reproducibility of the TCGA findings and includes gene expression measurements and survival times from 238 patients with HGSOC. In AOCS, we excluded patients with borderline tumors, endometrioid or other histology, missing vital status and grade 1 disease.

The TCGA data was downloaded from the cBio Portal (http://www.cbioportal.org/) using the R package $c g d s r$ and we used $z$-scores for the mRNA expression data. The AOCS data was downloaded from the GEO database (Accession number: GSE9891) and we applied RMA (using the Bioconductor package affy), which provides background 
correction, normalization, and summarization for gene expression measurements ${ }^{22}$. All the mRNA expression measures were centered and scaled to have unit variance according to standard practice. KEGG pathway annotations ${ }^{18}$ were downloaded from the MSigDB database (http://software.broadinstitute.org/gsea/msigdb). In this paper, we considered only the genes that are profiled in the KEGG database, belong to the previously reported 15 core signaling pathways in cancer ${ }^{23}$, and which are included in both the TCGA and AOCS datasets, which leaves a total of 1045 genes. These 15 core signaling pathways include known driver genes that appear in most cancer types and are proven regulators of core cellular processes including cell fate, cell survival, and genome maintenance ${ }^{24}$. The involvement of these pathways in ovarian cancer is well documented ${ }^{25-27}$.

\section{Methods}

InGRiD provides pathway-guided identification of patient subgroups based on gene expression measurements from patient tumors while utilizing patient survival information as the outcome variable. Let $\left(T_{i}, \delta_{i}\right)$ be the survival information (survival time and censoring indicator) of patient $i$, for $i=1, \ldots, n$, where $T_{i}$ is observed if $\delta_{i}=1$ and $T_{i}$ is

censored if $\delta_{i}=0$. Denote $\boldsymbol{x}_{\boldsymbol{i}}=\left(x_{i 1}, \ldots, x_{i p}\right)^{\prime}$ as the corresponding gene expression vector 
of patient $i$, where $n<p$. Let $m$ represent the number of pathways to which the $p$ genes belong. We consider the following proportional hazard model:

$h\left(T_{i}, \boldsymbol{x}_{\boldsymbol{i}}\right)=h_{0}\left(T_{i}\right) \exp \left\{\phi_{i}\right\}=h_{0}\left(T_{i}\right) \exp \left\{\sum_{r=1}^{m} \phi_{i r}\right\}$

where $\phi_{i r}$ relates the expression profile of pathway $r$ to the survival risk of patient $i$. The term $\phi_{\text {ir }}$ is given by

$\phi_{i r}=\boldsymbol{x}_{\boldsymbol{i}}^{\prime} \boldsymbol{U} \boldsymbol{W}_{\boldsymbol{r}} \boldsymbol{\beta}_{\boldsymbol{r}}=\left(x_{i 1}, \ldots, x_{i p}\right)\left(\begin{array}{ccc}u_{1} & \cdots & 0 \\ \vdots & \ddots & \vdots \\ 0 & \cdots & u_{p}\end{array}\right)\left(\begin{array}{ccc}w_{r 11} & \cdots & w_{r 1 K_{r}} \\ \vdots & \ddots & \vdots \\ w_{r p 1} & \cdots & w_{r p K_{r}}\end{array}\right)\left(\begin{array}{c}\beta_{r 1} \\ \vdots \\ \beta_{r K_{r}}\end{array}\right),($ eq1 $)$

where $\boldsymbol{U}$ is the $p \times p$ diagonal matrix of known gene-level weights, $\boldsymbol{W}_{r}$ is the $p \times K_{r}$ matrix summarizing the expression data of pathway $r$ as $K_{r}$ latent variables, and $\boldsymbol{\beta}_{\boldsymbol{r}}$ is the corresponding coefficient vector for the $K_{r}$ latent variables. By further defining the combined gene-level summarization matrix $\boldsymbol{W}=\left(\boldsymbol{W}_{1}, \cdots, \boldsymbol{W}_{m}\right)$ and the combined pathway-level coefficient vector $\boldsymbol{\beta}^{\prime}=\left(\boldsymbol{\beta}_{1}{ }^{\prime}, \cdots, \boldsymbol{\beta}_{m}{ }^{\prime}\right)^{\prime}$, this model can be further expressed as

$\phi_{i}=\sum_{r=1}^{m} \phi_{i r}=\sum_{r=1}^{m} \boldsymbol{x}_{\boldsymbol{i}}^{\prime} \boldsymbol{U} \boldsymbol{W}_{\boldsymbol{r}} \boldsymbol{\beta}_{\boldsymbol{r}}=\boldsymbol{x}_{\boldsymbol{i}}^{\prime} \boldsymbol{U} \sum_{r=1}^{m} \boldsymbol{W}_{\boldsymbol{r}} \boldsymbol{\beta}_{\boldsymbol{r}}=\boldsymbol{x}_{\boldsymbol{i}}^{\prime} \boldsymbol{U} \boldsymbol{W} \boldsymbol{\beta}$. (eq2) 
The $m$ pathways in (eq2) are not mutually exclusive because each of the $p$ genes can belong to more than one pathway. We use (eq2) as our base model to which further modifications can be made. We describe approaches to deal with the pathway overlapping issue in Section 3.1 while we estimate parameters in $\boldsymbol{W}$ and $\boldsymbol{\beta}$ sequentially, as described in Sections 3.3 and 3.4, respectively. We refer to our proposed approach as InGRiD hereafter and illustrate the entire workflow in Figure 1.

\subsection{Accounting for Overlapping Pathways}

In this subsection, we consider two different approaches for dealing with the pathway overlapping issue, namely the gene-cluster approach and the down-weight approach.

Consider the $p$ unique genes that have been mapped to the $m$ pathways based on a pathway annotation database such as KEGG. We denote the pathway membership of the $j$ th gene by the vector $\boldsymbol{z}_{\boldsymbol{j}}=\left(z_{j 1}, \ldots, z_{j m}\right)^{\prime}$, where $z_{j r}=1\{j$ th gene belongs to $r$ th pathway $\}$ for $r=$ $1, \ldots, m$. Finally, we denote the set of genes for the $r$ th gene set as $G_{r}$.

The gene-cluster approach assigns the $p$ genes into $m_{+}$gene sets $\left(m_{+}>m\right)$ according to the following rules: First, the $j$ th gene remains as a member of $G_{r}$ if it is a core member of the $r$ th pathway. The $j$ th gene is defined as a "core member" of the $r$ th

pathway if $z_{j r}=1$ and $z_{j r^{\prime}}=0$ for all $r^{\prime} \neq r$, i.e., it belongs to only the $r$ th pathway. 
Second, if a pathway has no core members, it will be dropped from further consideration. Let $m^{\prime}$ be the number of pathways with at least one core member and it follows that $m^{\prime} \leq$ $m$. Third, if the $j$ th gene is not a core member of any among the $m$ pathways (i.e., it maps to more than one pathway), then this gene is re-assigned to one of the gene sets $G_{m^{\prime}+1}, \ldots, G_{m_{+}}$based on the Partitioning Around Medoids (PAM) algorithm ${ }^{28}$. Let $d\left(\mathbf{z}_{\boldsymbol{j}}, \mathbf{z}_{\boldsymbol{j}}\right)$ be the binary distance between genes $j$ and $j^{\prime}$ located in cluster $c$. The PAM algorithm groups genes into $k$ clusters by minimizing the within-cluster distance $\sum_{c=1}^{k} \sum_{C(j)=c\left(j^{\prime}\right)=c} d\left(\mathbf{z}_{\boldsymbol{j}}, \mathbf{z}_{\boldsymbol{j}^{\prime}}\right)$, where $C($.$) indicates the cluster membership of gene j$. Hence, by re-clustering the $p$ genes into the $m_{+}$gene sets, the gene-cluster approach creates a unique membership for each gene.

Unlike the $k$-means approach which minimizes within-cluster distances with respect to cluster means, the PAM approach assigns cluster memberships based on the closeness of genes to a representative gene of each cluster. In order to determine the optimal number of clusters $k$, we utilize the silhouette width $(s w)$, which measures the quality of the assignment of an individual element to its assigned cluster. A gene is considered to be well clustered if $s w \approx 1$, whereas a gene is considered to be assigned to a wrong cluster if $s w<0$. The optimal choice of $k$ depends on both the genomic data at 
hand and the pathway annotations considered. In our data analysis, we chose $k=4$ by comparing the mean $s w$ across $k=1, \ldots, 10$. We implemented PAM based on the build and swap algorithms using the R package cluster.

As an alternative to the gene-cluster approach, we also consider down-weighting overlapping genes. Let $f_{j}$ be the number of occurrences of gene $j$ across all the pathways, i.e., $f_{j}=\sum_{r=1}^{m} z_{j r}$. The down-weighting approach ${ }^{20}$ determines the weight of gene $j$ as

$$
u_{j}=1+\sqrt{\frac{\max _{\mathrm{j} \prime}\left(f_{j^{\prime}}\right)-f_{j}}{\max _{\mathrm{j} \prime}\left(f_{j^{\prime}}\right)-\min _{\mathrm{j} \prime}\left(f_{j^{\prime}}\right)}} \cdot(\text { eq3) }
$$

Note that the weight $u_{j}$ is a monotonically decreasing function of $f_{j}$. Specifically, $u_{j}=1$ if a gene appears in all the $m$ pathways whereas $u_{j}=2$ if it belongs to only a single pathway.

Using the notations of (eq1) and (eq2), the approaches described above can be summarized as follows. In the gene-cluster approach, the size of matrix $\boldsymbol{W}$ in (eq2) now becomes $p \times \sum_{r=1}^{m_{+}} K_{r}$ (which corresponds to $p \times \sum_{r=1}^{m+4} K_{r}$ in our real data analysis) instead of $p \times \sum_{r=1}^{m} K_{r}$ while $\boldsymbol{U}$ in (eq2) is set to be an identity matrix. In the down-weighting approach, the size of matrix $\boldsymbol{W}$ is $p \times \sum_{r=1}^{m} K_{r}$ as in (eq2) and the $j$ th diagonal element of $\boldsymbol{U}$ is set to $u_{j}$ given in (eq3). As a baseline, we also consider a version of InGRiD without 
accounting for the pathway overlap issue. Here, $\boldsymbol{U}$ is again set to be an identity matrix and the size of matrix $\boldsymbol{W}$ still remains as $p \times \sum_{r=1}^{m} K_{r}$. In the remainder of this paper, we refer to InGRiD based on the gene-cluster approach, the down-weighting approach, and without accounting for the pathway overlap issue as gcInGRiD, dwInGRiD and naïve InGRiD, respectively.

\subsection{Gene Pre-filtering}

To improve the signal-to-noise ratio and estimation stability, we first eliminate the most unlikely gene predictors by conducting a supervised pre-filtering using a Cox model applied to each gene separately and including only the genes with $p$-value $<0.5$ for further analysis. The performance of InGRiD is not sensitive to the choice of p-value cutoff point as long as we are not overly aggressive in eliminating genes in the pre-filtering step (e.g.,

$p$-value $<0.2$ ). Specifically, different $\mathrm{p}$-value cutoff points led to similar results in terms of molecular feature selection (Supplemental Tables 1 and 2) and survival-guided patient subgroup identification (Supplemental Figure 1).

\subsection{Summarizing Gene Set Expression Profiles using a SPLS Cox Regression}


In order to effectively construct the summarization matrices $\boldsymbol{W}_{r}$ in (eq1) by taking into account correlation among the genes in each gene set, we utilize a SPLS Cox regression model ${ }^{29}$. The SPLS Cox model calculates deviance residuals from a Cox model without covariates and fits a SPLS regression model using deviance residuals as responses. SPLS regression was developed on the basis of partial least squares (PLS), which seeks direction vectors that do not only capture a large proportion of predictor variance but also explains a large proportion of association with responses. On the other hand, SPLS has an important distinction compared to PLS. Specifically, while PLS assumes that all the genes in $G_{r}$ are associated with the survival outcome (i.e., all the elements in direction vectors are not zeros), SPLS sequentially constructs each direction vector only based on "important" genes by shrinking some of its elements to zero. Hence, SPLS provides simultaneous gene selection and dimension reduction. SPLS has been reported to have better predictive performance than PLS, especially in the context of genomic studies ${ }^{30,31}$.

We estimate each $\boldsymbol{W}_{r}$ in (eq1) by fitting a SPLS Cox regression model of patient survival on gene expression measurements separately for each gene set $G_{r}$. As a result, if gene $j$ is not in $G_{r}$, all the elements in the $j$ th row of $\boldsymbol{W}_{r}$ are zeros. Otherwise, the rows of $\boldsymbol{W}_{r}$ corresponding to the genes belonging to $G_{r}$ are estimated using the SPLS Cox regression. This step significantly simplifies the pathway-level analysis described in detail 
in the next subsection because the length of $\boldsymbol{x}_{\boldsymbol{i}}^{\prime} \boldsymbol{U} \boldsymbol{W}$ in (eq2) is usually much smaller than the length of the original gene expression data $\boldsymbol{x}_{\boldsymbol{i}}^{\prime}$, i.e., $\sum_{r=1} K_{r} \ll \mathrm{p}$. Note that in gcInGRiD, there is no overlap between gene sets and as a result, there are at most $K_{r}$ nonzero elements for the row corresponding to a gene belonging to $G_{r}$ in the estimated combined summarization matrix $\widehat{\boldsymbol{W}}$ in (eq2). However, this is not the case for dwInGRiD and naïve InGRiD because they allow overlap between gene sets. We utilized the $\mathrm{R}$ package plsRcox as an implementation of the SPLS Cox regression.

In our data analysis, we set $K_{r}=1$ for all $r$ and determine only the optimal shrinkage parameter of SPLS for each gene set $G_{r}$ using 10-fold cross validation. Fixing $K_{r}=1$ significantly reduces the complexity of cross validation. In addition, fixing $K_{r}=1$ makes each $\boldsymbol{W}_{r}$ in (eq1) a length $p$ column vector instead of a $p \times K_{r}$ matrix. In this case, (eq2) can be further simplified as

$$
\phi_{i}=\left(x_{i 1}, \ldots, x_{i p}\right)\left(\begin{array}{ccc}
u_{1} & \cdots & 0 \\
\vdots & \ddots & \vdots \\
0 & \cdots & u_{p}
\end{array}\right)\left(\begin{array}{ccc}
w_{111} & \cdots & w_{m 11} \\
\vdots & \ddots & \vdots \\
w_{1 p 1} & \cdots & w_{m p 1}
\end{array}\right)\left(\begin{array}{c}
\beta_{11} \\
\vdots \\
\beta_{m 1}
\end{array}\right)=\sum_{r=1}^{m} \sum_{j=1}^{p} x_{i j} u_{j} w_{r j 1} \beta_{r 1} .
$$

Put another way, $\phi_{i r}=\sum_{j=1}^{p} x_{i j} u_{j} w_{r j 1} \beta_{r 1}$. Hence, InGRiD essentially determines the contribution of each gene in $G_{r}$ to the patient- and pathway-specific hazards $\phi_{i r}$ based on its gene-level contribution to the latent variables summarizing gene set expression data 
$\left(w_{r j 1}\right)$ adjusted by its corresponding weight $\left(u_{j}\right)$ and its corresponding pathway-level effect $\left(\beta_{r 1}\right)$.

Alternatively, both $K_{r}$ and the shrinkage parameter can be determined using 10fold cross validation. Supplemental Table 3 tabulates the top genes in each selected gene set by gcInGRiD with $K_{r}$ not fixed and Supplemental Figure 2 shows the identified patient subgroups by gcInGRiD with $K_{r}$ not fixed. These results indicate that fixing $K_{r}=$ 1 has little impact on the molecular feature selection (Table 1) and patient subgroup identification of gcInGRiD (Figure 2).

\subsection{Pathway-level Analysis using a LASSO-Penalized Cox Regression}

Given the estimated summarization matrix $\widehat{\boldsymbol{W}}$, we now identify a parsimonious set of pathways associated with patient survival. Specifically, we fit a LASSO-penalized Cox regression ${ }^{32}$ on latent variables $\left(\boldsymbol{x}_{\boldsymbol{i}}^{\prime} \boldsymbol{U} \widehat{\boldsymbol{W}}\right)$ derived from all the gene sets by minimizing the objective function $-l(\boldsymbol{\beta})+\lambda\|\boldsymbol{\beta}\|_{1}$ with respect to $\boldsymbol{\beta}$, where $l(\boldsymbol{\beta})$ is the partial likelihood function and $\lambda$ is a tuning parameter controlling the amount of shrinkage, which is again determined using 10-fold cross validation. We utilized the R package glmnet as an implementation of the LASSO-penalized Cox regression. 


\subsection{Patient Subgroup Identification}

We identify patient subgroups by clustering patients based on the degree of activation of their "risk pathways". First, we calculate the patient- and pathway-specific hazards $\hat{\phi}_{i r}=$ $\boldsymbol{x}_{\boldsymbol{i}}^{\prime} \boldsymbol{U} \widehat{\boldsymbol{W}}_{\boldsymbol{r}} \widehat{\boldsymbol{\beta}}_{\boldsymbol{r}}$ defined in (eq1). Then, we consider the gene set $G_{r}$ as a "risk pathway" for

patient $i$ if $\hat{\phi}_{i r}>0$. Finally, a patient is categorized as "low-risk group" if $\sum_{r} 1\left\{\hat{\phi}_{i r}>0\right\}$ is less than the first quartile, as "intermediate-risk group" if $\sum_{r} 1\left\{\widehat{\phi}_{i r}>0\right\}$ is between the first and third quartiles, and as "high-risk group" if $\sum_{r} 1\left\{\hat{\phi}_{i r}>0\right\}$ is higher than the third quartile.

\section{Results}

We first summarize the pathways and genes identified by InGRiD in Section 4.1 while patient subgroups identified by InGRiD are presented in Section 4.2 based on the TCGA dataset ${ }^{1}$. The predictive performance of InGRiD is evaluated using the TCGA dataset ${ }^{1}$ as internal validation (Section 4.3) and the AOCS data ${ }^{21}$ as external validation (Section 4.4). In Section 4.5, we compare the pathway-level analysis with the gene-level analysis in the sense of robustness in the identification of pathways/genes and patient subgroups. In addition to InGRiD, we also consider the gene index count model that performs LASSO- 
penalized Cox regression at the gene level after pre-filtering and then categorizes prognostic risks of each individual patient based on the count of genes associated with increased hazards using a procedure similar to those described in Section 3.5.

\subsection{Pathway and Gene Selection}

We summarize pathway and gene selection results of gcInGRiD in Table 1, which shows the selected gene sets, the numbers of total and selected genes in each gene set, the geneset-level LASSO coefficient estimates, and the top three genes in each gene set. Gene sets are ranked based on their LASSO coefficient estimates. The gene-cluster approach of gcInGRiD identified four additional gene sets, namely "MAPK\&APOPTOSIS", "DNAREPAIR", "WNT\&HEDGEHOG", and "TGF_BETA\&CELL_CYCLE\&WNT" (the list of genes belonging to each of these additional gene sets is provided in Supplemental Table 4). gcInGRiD identified 357 unique genes from 13 gene sets using the TCGA data, where these 13 gene sets include eleven KEGG pathways comprising only core genes and two gene sets generated from common genes shared across KEGG pathways

(“MAPK\&APOPTOSIS" and "DNA-REPAIR").

First, the "MAPK\&APOPTOSIS" cluster predominantly consists of genes from the MAPK (56 genes) and the APOPTOSIS pathways (41 genes), while other contributors to 
this cluster include PHOSPHATIDYLINOSITOL_SIGNALING (11 genes),

CELL_CYCLE (6 genes), MTOR_SIGNALING (16 genes), WNT_SIGNALING (23

genes), HEDGEHOG_SIGNALING (3 genes), TGF_BETA_SIGNALING (5 genes), and JAK_STAT_SIGNALING (17 genes). Among the 37 cancer genes selected from "MAPK\&APOPTOSIS", 32 genes are from the MAPK pathway, 20 are from the APOPTOSIS pathway, 11 are from the WNT_SIGNALING pathway, and 8 are from the MTOR_SIGNALING pathway. Therefore, "MAPK\&APOPTOSIS” mainly represents cross talk between MAPK and other cancer pathways.

Second, most genes in the "DNA-REPAIR" cluster are from pathways involved in DNA repair including BASE_EXCISION_REPAIR (12 genes), NUCLEOTIDE_ EXCISION_REPAIR (20 genes) and MISMATCH_REPAIR (14 genes). A few genes in the "DNA-REPAIR" cluster are from the NON_HOMOLOGOUS_END_JOINING (2 genes) and CELL_CYCLE (3 genes) pathways. The three genes selected from the "DNAREPAIR" cluster are contributed by the BASE_EXCISION_REPAIR (3 genes), MISMATCH_REPAIR (2 genes), NUCLEOTIDE_EXCISION_REPAIR (2 genes), and NON_HOMOLOGOUS_END_JOINING (1 gene) pathways.

Two other clusters of overlapping genes are not selected by gcInGRiD. The gene set "WNT\&HEDGEHOG" mostly consists of the genes from the WNT_SIGNALING (31 
genes) and the HEDGEHOG_SIGNALING (28 genes) pathways, with a few genes from the PHOSPHATIDYLINOSITOL_SIGNALING_SYSTEM (4 genes), CELL_CYCLE (1 gene), NOTCH_SIGNALING (6 genes) and TGF_BETA_SIGNALING (7 genes) pathways. Genes comprising the "TGF_BETA\&CELL_CYCLE\&WNT" cluster are mostly from the TGF_BETA_SIGNALING (28 genes), CELL_CYCLE (24 genes) and WNT_SIGNALING (19 genes) pathways.

Naïve InGRiD, which has no special considerations for overlapping pathways, selected twelve cancer pathways (Table 2), which are similar to those selected by gcInGRiD. However, not accounting for pathway overlapping created significant difficulties in interpreting the findings from naïve InGRiD. First, it is not clear whether a pathway is selected by naïve InGRiD because of its independent association with survival or due to the genes that belong to other pathways that are actually associated with survival. In contrast, gcInGRiD facilitates a better understanding on the biological processes underlying cancer by separating out the independent effect of a pathway from the cross talk among pathways. Second, it is also hard to interpret top-ranking genes selected by naïve InGRiD because sometimes these top-ranking genes appear in multiple selected pathways and coefficient estimates for these genes are different across different pathways. In contrast, gcInGRiD does not have this issue because each gene can appear only in one gene set. 
Interestingly, dwInGRiD selected the same pathways and genes as the naïve approach (Table 3). This indicates that although down-weighting common genes reduces the similarities among pathways, it still does not fully resolve the overlap among pathways. Due to the increased interpretability of gcInGRiD compared to dwInGRiD and naïve InGRiD, we focus on gcInGRiD in the following subsections.

\subsection{Patient Subgroup Identification}

After identifying a parsimonious set of cancer-associated pathways, gcInGRiD categorizes patients by counting "activated risk pathways" $\left(\hat{\phi}_{i r}>0\right)$ for each patient. Figure 2 shows the observed survival curves for patient subgroups identified by gcInGRiD and the gene index count approach. In gcInGRiD, the low-risk group had 4 or fewer activated risk pathways, whereas the high-risk group has a minimum of 9 activated risk pathways. These cut points corresponded to the 25 and 75 percentiles of the risk pathway counts. The $\log$ rank test revealed highly significant differences between groups: high versus intermediate risk groups $(\mathrm{p}=3 \mathrm{e}-5)$ and intermediate versus low risk groups $(\mathrm{p}=5 \mathrm{e}-5)$. The median survival times for the high, intermediate and low risk groups were 31.6 months (95\% CI: 27.6 - 38.4), 41.5 months (95\% CI: 38.0 - 47.7) and 70.8 months (95\% CI: $51.9-90.1)$, respectively. dwInGRiD and naïve InGRiD performed similarly as gcInGRiD (data not 
shown). The gene index count model is also capable of identifying patient subgroups, where the median survival times of the high, intermediate and low risk groups were 32.0 months (95\% CI: 29.0 - 35.4), 44.9 months (95\% CI: 38.3 - 51.3) and 64.0 months (95\% CI: $52.0-76.9)$, respectively.

\subsection{Internal Validation by Partitioning the TCGA Dataset}

To further compare the pathway-level (gcInGRiD) and the gene-level (gene index count model) approaches, we conducted internal validation by randomly partitioning the TCGA data into training $(n=243)$ and test data $(n=242) 100$ times. At each iteration, we fit the InGRiD and the gene index count model using the training data and evaluate their performances in predicting patient subgroups using the test data. Figure $\mathbf{3}$ shows that gcInGRiD has better predictive performance compared to the gene index count model. Specifically, the time-dependent AUC at 5 years is $0.59(\mathrm{SD}=0.06)$ and $0.49(\mathrm{SD}=0.06)$ for gcInGRiD and the gene index model, respectively ( $\mathrm{p}<0.001, t$-test). Comparisons at other time points produces similar results. gcInGRiD is also more likely to separate patient subgroups based on their predicted prognostic risks. The $-\log 10$ transformed $p$-values of a global $F$-test were $1.65(\mathrm{SD}=1.08)$ and $0.41(\mathrm{SD}=0.44)$ for the pathway-level and the genelevel analyses, respectively ( $<<0.001, t$-test). The patient subgroups identified by 
gcInGRiD are also more distinct from each other in terms of restricted mean death time, which is the time a patient is expected to live during the 60 months of follow-up. When the "subgroup distance" is defined as the difference in restricted mean times between the high and the low risk groups, the subgroup distance is $6.0(\mathrm{SD}=4.1)$ months and $-0.4(\mathrm{SD}=3.8)$ months for the pathway-level and the gene-level analyses, respectively ( $<<0.001, t$-test).

\subsection{External Validation in an Independent Dataset}

The performance of the pathway-level (gcInGRiD) and the gene-level (gene index count model) approaches were further evaluated using the independent AOCS data. Specifically, for gcInGRiD, the patient- and pathway-specific hazards $\left(\hat{\phi}_{i r}\right)$ were calculated for new observations in the AOCS data using the InGRiD parameters $\left(\widehat{\boldsymbol{W}}_{\boldsymbol{r}}\right.$ and $\left.\widehat{\boldsymbol{\beta}}_{\boldsymbol{r}}\right)$ learned from the TCGA data. For the gene index count model, the cancer-related genes identified using the TCGA data were used to predict the prognostic risks of patients in the AOCS data. Figure 4 shows the Kaplan-Meier curves of predicted patient subgroups using the AOCS data. The gcInGRiD performed best at identifying patient subgroups ( $\mathrm{p}=0.00061$, global $F$ test) compared to dwInGRiD ( $\mathrm{p}=0.0415$, global $F$-test), naïve InGRiD ( $\mathrm{p}=0.0478$, global $F$-test), and the gene-index count model (p=0.00841, global $F$-test). 


\subsection{Stability Analysis}

Finally, we compared the stability in identifying cancer-associated molecular features and patient subgroups between the pathway-level (gcInGRiD) and the gene-level (gene index count model) approaches using the TCGA data (Figure 5). To test the stability in feature selection, we randomly sampled $50 \%(\mathrm{n}=242)$ of the TCGA patients 100 times and evaluated the percentage of the molecular features that can still be identified using these subsamples. The pathway-level analysis showed significantly higher reproducibility in feature selection than the gene-level analysis $(\mathrm{P}<0.001, t$-test). Specifically, among the gene sets selected by gcInGRiD (Table 1), 64\% ( $\mathrm{SD}=15 \%$ ) of them could be reproduced. In contrast, only $15 \%(\mathrm{SD}=14 \%$ ) of the top-ranking genes selected by the gene index count model could be reproduced. Note that the gene index count model failed to select any genes $30 \%$ of the time, which was not the case for the pathway-level analysis.

To test the stability in subgroup identification, we randomly sampled $80 \%$ of the genes from the TCGA data 100 times and examined the percentage of patients that can still be assigned to the same subgroups identified using the complete TCGA data. The pathway-level analysis was also significantly more reproducible in identifying patient subgroups ( $(\mathrm{P}<0.001, t$-test), where $78 \%(\mathrm{SD}=4 \%)$ and $71 \%(\mathrm{SD}=8 \%)$ of patients were 
assigned to the same risk groups in gcInGRiD and the gene index count model, respectively.

\section{Discussion}

In this paper, we present InGRiD, a statistical framework for the simultaneous identification of cancer patient subgroups and key molecular features. InGRiD focuses on improving the robustness of biological findings and their interpretations by utilizing the pathway information and taking into account the pathway overlap issue. The application of InGRiD to the TCGA HGSOC data showed that InGRiD can effectively classify patients according to differences in survival probabilities and this risk group classification could be further reproduced in an external dataset (AOCS data). In addition, InGRiD with the genecluster approach (gcInGRiD) did not only identify key cancer pathways (e.g., MAPK and HEDGEHOG_SIGNALLING), but could also distinguish the interaction among several

pathways from the independent effect of an individual pathway. Since tumors can develop resistance to therapies targeting a single pathway, the development of novel statistical approaches for identifying the interactions among pathways also provides insight into the development of anti-tumor agents that target multiple pathways involved in cancer. We 
further demonstrated the superior performance of the pathway-level analysis in selecting key molecular features and identifying patient subgroups compared to the gene-level analysis based on internal and external validations. Moreover, the pathway-level analysis proved to be more robust than the gene-level analysis in the sense that 1) the pathway-level analysis is more likely to identify similar features across datasets consisting of different patients; and 2) the pathway-level analysis is also more likely to assign a patient to the same subgroup upon potential perturbation of molecular features.

We are currently also working on improving InGRiD further by pursuing the following directions. First, in this paper, we considered only the gene expression data. While it is often reported that gene expression data are sufficiently informative for predicting patient risk, we also plan to utilize and integrate other genomic data types, such as copy number alterations, DNA methylation, and somatic mutations. This can potentially improve statistical power in patient risk prediction and also facilitate understanding of biological mechanisms associated with cancer progression. Second, the patient subgroups identified by InGRiD are currently based on differences in prognostic risks. In addition, we are also working on the classification of patients based on the weighted combination of selected pathways. Third, in this paper, we conservatively focused on the KEGG pathway annotation because the KEGG database is based on human curation and has been reported 
to be of high quality and stability ${ }^{18}$. We plan to consider other pathway databases such as Reactome ${ }^{33}$, WikiPathways ${ }^{34}$, and BioPortal Pathway Ontology ${ }^{35}$. This can exploit larger gene sets in the prediction model and also improve the quality of gene annotations. We expect that InGRiD will be a powerful approach to investigate cancer subtypes according to their molecular features and irrespective of the tumor anatomical site by identifying shared pathogeneses, which will offer opportunities for overlapping treatments across various cancer subtypes. 


\section{Declaration of conflicting interests}

The author(s) declared no potential conflicts of interest with respect to the research, authorship, and/or publication of this article.

\section{Funding}

DC, LEK, GH, AL and ZS were partially supported by the NIH/NCI grant (R21

CA209848). DC, GH and AL were also partially supported by the NIH/NIGMS grant (R01 GM122078). 


\section{References}

1. Cancer Genome Atlas Network. Integrated genomic analyses of ovarian carcinoma. Nature. 2011; 474: 609-15.

2. Cancer Genome Atlas Network. Comprehensive molecular characterization of human colon and rectal cancer. Nature. 2012; 487: 330-7.

3. Cancer Genome Atlas Research Network. Comprehensive molecular characterization of gastric adenocarcinoma. Nature. 2014; 513: 202-9.

4. Hoadley KA, Yau C, Wolf DM, et al. Multiplatform analysis of 12 cancer types reveals molecular classification within and across tissues of origin. Cell. 2014; 158: 929-44. 5. Kandoth C, Schultz N, Cherniack AD, et al. Integrated genomic characterization of endometrial carcinoma. Nature. 2013; 497: 67-73.

6. Cancer Genome Atlas Network. Comprehensive molecular portraits of human breast tumours. Nature. 2012; 490: 61-70.

7. Mo Q, Wang S, Seshan VE, et al. Pattern discovery and cancer gene identification in integrated cancer genomic data. Proc Natl Acad Sci U S A. 2013; 110: 4245-50.

8. Tyekucheva S, Marchionni L, Karchin R and Parmigiani G. Integrating diverse genomic data using gene sets. Genome Biol. 2011; 12: R105.

9. Vaske CJ, Benz SC, Sanborn JZ, et al. Inference of patient-specific pathway activities from multi-dimensional cancer genomics data using PARADIGM.

Bioinformatics. 2010; 26: i237-45.

10. Shen R, Olshen AB and Ladanyi M. Integrative clustering of multiple genomic data types using a joint latent variable model with application to breast and lung cancer subtype analysis. Bioinformatics. 2009; 25: 2906-12.

11. Shen R, Wang S and Mo Q. Sparse integrative clustering of multiple omics data sets. Ann Appl Stat. 2013; 7: 269-94.

12. Glaab E. Using prior knowledge from cellular pathways and molecular networks for diagnostic specimen classification. Brief Bioinform. 2015.

13. Kim S, Kon M and DeLisi C. Pathway-based classification of cancer subtypes. Biol Direct. 2012; 7: 21.

14. Chang YH, Chen CM, Chen HY and Yang PC. Pathway-based gene signatures predicting clinical outcome of lung adenocarcinoma. Sci Rep. 2015; 5: 10979.

15. Allison DB, Cui X, Page GP and Sabripour M. Microarray data analysis: from disarray to consolidation and consensus. Nat Rev Genet. 2006; 7: 55-65. 
16. Hofree M, Shen JP, Carter H, Gross A and Ideker T. Network-based stratification of tumor mutations. Nat Methods. 2013; 10: 1108-15.

17. Eng KH, Wang S, Bradley WH, Rader JS and Kendziorski C. Pathway index models for construction of patient-specific risk profiles. Stat Med. 2013; 32: 1524-35.

18. Kanehisa M and Goto S. KEGG: Kyoto encyclopedia of genes and genomes.

Nucleic Acids Res. 2000; 28: 27-30.

19. Jiang Z and Gentleman R. Extensions to gene set enrichment. Bioinformatics. 2007; 23: 306-13.

20. Tarca AL, Draghici S, Bhatti G and Romero R. Down-weighting overlapping genes improves gene set analysis. BMC Bioinformatics. 2012; 13: 136.

21. Tothill RW, Tinker AV, George J, et al. Novel molecular subtypes of serous and endometrioid ovarian cancer linked to clinical outcome. Clin Cancer Res. 2008; 14: 5198208.

22. Bolstad BM, Irizarry RA, Astrand M and Speed TP. A comparison of normalization methods for high density oligonucleotide array data based on variance and bias. Bioinformatics. 2003; 19: 185-93.

23. Jones S, Zhang X, Parsons DW, et al. Core signaling pathways in human pancreatic cancers revealed by global genomic analyses. Science. 2008; 321: 1801-6.

24. Vogelstein B, Papadopoulos N, Velculescu VE, Zhou S, Diaz LA, Jr. and Kinzler KW. Cancer genome landscapes. Science. 2013; 339: 1546-58.

25. Winterhoff BJ, Maile M, Mitra AK, et al. Single cell sequencing reveals heterogeneity within ovarian cancer epithelium and cancer associated stromal cells. Gynecol Oncol. 2017.

26. Scarbrough PM, Weber RP, Iversen ES, et al. A cross-cancer genetic association analysis of the DNA repair and DNA damage signaling pathways for lung, ovary, prostate, breast, and colorectal cancer. Cancer Epidemiol Biomarkers Prev. 2016; 25: 193-200.

27. Verhaak RG, Tamayo P, Yang JY, et al. Prognostically relevant gene signatures of high-grade serous ovarian carcinoma. J of Clin Invest. 2013; 123: 517-25.

28. Reynolds AP, Richards G, de la Iglesia B and Rayward-Smith VJ. Clustering rules: a comparison of partitioning and hierarchical clustering algorithms. J Math Model Algorithm. 2006; 5: 475-504.

29. Bastien P, Bertrand F, Meyer N and Maumy-Bertrand M. Deviance residuals-based sparse PLS and sparse kernel PLS regression for censored data. Bioinformatics. 2015; 31: 397-404. 
30. Chun $\mathrm{H}$ and Keles S. Sparse partial least squares regression for simultaneous dimension reduction and variable selection. J R Stat Soc Series B Stat Methodol. 2010; 72: 3-25.

31. Chung D and Keles S. Sparse partial least squares classification for high dimensional data. Stat Appl Genet Mol Biol. 2010; 9: Article17.

32. Tibshirani R. The lasso method for variable selection in the Cox model. Stat Med. 1997; 16: 385-95.

33. Croft D, O'Kelly G, Wu G, et al. Reactome: a database of reactions, pathways and biological processes. Nucleic Acids Res. 2011; 39: D691-7.

34. Kutmon M, Riutta A, Nunes N, et al. WikiPathways: capturing the full diversity of pathway knowledge. Nucleic Acids Res. 2016; 44: D488-94.

35. Whetzel PL, Noy NF, Shah NH, et al. BioPortal: enhanced functionality via new Web services from the National Center for Biomedical Ontology to access and use ontologies in software applications. Nucleic Acids Res. 2011; 39: W541-5. 


\section{Table 1. Top gene sets and genes selected by gcInGRiD based on the gene-cluster}

approach. Gene sets are ranked based on their gene-set-level LASSO coefficient estimates.

Gene sets consisting of common genes across pathways are written in bold face. The number within parenthesis in the column 'Genes Selected' refers to the total number of genes in each gene set.

\begin{tabular}{|c|c|c|c|c|c|}
\hline Gene Sets Selected & $\begin{array}{l}\text { Genes } \\
\text { Selected }\end{array}$ & Gene Set & \multicolumn{3}{|c|}{ Top Three Genes } \\
\hline HEDGEHOG_SIGNALING & $12(20)$ & 0.167 & CSNKIG3 & GAS1 & CSNK1D \\
\hline MAPK\&APOPTOSIS & $37(68)$ & 0.093 & PPP3CA & RPS6KA2 & $T G F B R I$ \\
\hline NUCLEOTIDE_EXCISION_REPAIR & 2(19) & 0.072 & GTF2H4 & $D D B 2$ & \\
\hline CELL_ADHESION_MOLECULES_CAMS & $59(103)$ & 0.065 & CD6 & CTLA4 & CLDN6 \\
\hline NOTCH_SIGNALING & $1(36)$ & 0.062 & NOTCH4 & & \\
\hline PHOSPHATIDYLINOSITOL_SIGNALING & $30(58)$ & 0.06 & PLCG1 & $P I P 4 K 2 B$ & IMPA2 \\
\hline CELL_CYCLE & $2(84)$ & 0.059 & $M C M 3$ & ANAPC11 & \\
\hline MISMATCH_REPAIR & $1(7)$ & 0.045 & $S S B P 1$ & & \\
\hline JAK_STAT_SIGNALING & $67(115)$ & 0.039 & SOCS5 & $I L 21 R$ & IFNA21 \\
\hline WNT_SIGNALING & $39(67)$ & 0.016 & $A P C$ & CSNK2A2 & FZD1 \\
\hline BASE_EXCISION_REPAIR & $10(20)$ & 0.011 & MUTYH & $U N G$ & APEX1 \\
\hline MAPK_SIGNALING & $94(189)$ & 0.01 & $P L A 2 G 2 D$ & PDGFRA & $F G F 7$ \\
\hline DNA-REPAIR & $3(22)$ & 0.005 & POLD2 & FEN1 & POLD3 \\
\hline WNT\&HEDGEHOG & $0(38)$ & 0 & & & \\
\hline TGF_BETA\&CELL_CYCLE\&WNT & $0(34)$ & 0 & & & \\
\hline NON_HOMOLOGOUS_END_JOINING & $0(10)$ & 0 & & & \\
\hline MTOR_SIGNALING & $0(29)$ & 0 & & & \\
\hline APOPTOSIS & $0(39)$ & 0 & & & \\
\hline TGF_BETA_SIGNALING & $0(43)$ & 0 & & & \\
\hline
\end{tabular}


Table 2. Top pathways and genes selected by naïve InGRiD which makes no considerations for the pathway overlap issue. Pathways are ranked based on their pathway-level LASSO coefficient estimates. The number within parenthesis in the column 'Genes Selected' refers to the total number of genes in each gene set.

\begin{tabular}{|c|c|c|c|c|c|}
\hline Pathways Selected & Genes & Pathway & \multicolumn{3}{|c|}{ Top Three Genes } \\
\hline HEDGEHOG_SIGNALING & $29(56)$ & 0.117 & CSNK1G3 & GAS1 & $B T R C$ \\
\hline MTOR_SIGNALING & $1(52)$ & 0.089 & RPS6KA2 & & \\
\hline NOTCH_SIGNALING & $1(47)$ & 0.08 & NOTCH4 & & \\
\hline NUCLEOTIDE_EXCISION_REPAIR & $4(44)$ & 0.064 & POLD2 & GTF2H4 & $D D B 2$ \\
\hline CELL_CYCLE & $2(128)$ & 0.058 & MCM3 & ANAPC11 & \\
\hline CELL_ADHESION_MOLECULES_CAMS & $59(134)$ & 0.057 & CD6 & CTLA4 & CLDN6 \\
\hline JAK_STAT_SIGNALING & $80(155)$ & 0.052 & SOCS5 & $I L 21 R$ & IFNA21 \\
\hline PHOSPHATIDYLINOSITOL_SIGNALING & $34(76)$ & 0.051 & $P L C G 1$ & $P I P 4 K 2 B$ & IMPA2 \\
\hline MAPK_SIGNALING & $127(267)$ & 0.04 & $P L A 2 G 2 D$ & PPP $3 C A$ & RPS6KA2 \\
\hline MISMATCH_REPAIR & $3(23)$ & 0.026 & POLD2 & $S S B P 1$ & POLD3 \\
\hline APOPTOSIS & $1(88)$ & 0.014 & PPP $3 C A$ & & \\
\hline WNT_SIGNALING & $75(151)$ & 0.001 & PPP $3 C A$ & $A P C$ & CSNK2A2 \\
\hline BASE_EXCISION_REPAIR & $0(35)$ & 0 & & & \\
\hline NON_HOMOLOGOUS_END_JOINING & $0(14)$ & 0 & & & \\
\hline TGF_BETA_SIGNALING & $0(86)$ & 0 & & & \\
\hline
\end{tabular}




\section{Table 3. Top pathways and genes selected by dwInGRiD based on the down-}

weighting approach. Pathways are ranked based on their pathway-level LASSO

coefficient estimates. The number within parenthesis in the column 'Genes Selected' refers

to the total number of genes in each gene set.

\begin{tabular}{l|c|c|lcc}
\hline \multicolumn{1}{c|}{ Pathways Selected } & $\begin{array}{c}\text { Genes } \\
\text { Selected }\end{array}$ & $\begin{array}{c}\text { Pathway } \\
\text { Coefficient }\end{array}$ & \multicolumn{3}{|c}{ Top Three Genes } \\
\hline HEDGEHOG_SIGNALING & $29(56)$ & 0.059 & CSNK1G3 & GAS1 & BTRC \\
MTOR_SIGNALING & $1(52)$ & 0.047 & RPS6KA2 & & \\
NOTCH_SIGNALING & $1(47)$ & 0.038 & NOTCH4 & & POLD2 \\
NUCLEOTIDE_EXCISION_REPAIR & $4(44)$ & 0.035 & GTF2H4 & DDB2 & IMPA2 \\
PHOSPHATIDYLINOSITOL_SIGNALING & $34(76)$ & 0.028 & PLCG1 & PIP4K2B & CLDN6 \\
CELL_CYCLE & $2(128)$ & 0.028 & MCM3 & ANAPC11 & IFNA21 \\
CELL_ADHESION_MOLECULES_CAMS & $59(134)$ & 0.028 & CD6 & CTLA4 & PDGFRA \\
JAK_STAT_SIGNALING & $80(155)$ & 0.027 & SOCS5 & IL21R & POLD3 \\
MAPK_SIGNALING & $127(267)$ & 0.017 & PLA2G2D & RPS6KA2 & PDG \\
MISMATCH_REPAIR & $3(23)$ & 0.016 & SSBP1 & POLD2 & \\
APOPTOSIS & $1(88)$ & 0.007 & PPP3CA & & CSNK2A2 \\
WNT_SIGNALING & $75(151)$ & 0.004 & APC & PPP3CA & \\
BASE_EXCISION_REPAIR & $0(35)$ & 0 & & & \\
NON_HOMOLOGOUS_END_JOINING & $0(14)$ & 0 & & & \\
TGF_BETA_SIGNALING & $0(86)$ & 0 & & & \\
\hline
\end{tabular}


Figure 1. Workflow of the proposed InGRiD approach.

Figure 2. Kaplan-Meier curves for patient subgroups identified by the pathway-level analysis (gcInGRiD) and the gene-level analysis (gene index count model) using the TCGA high-grade serous ovarian cancer data. Patient subgroups are color-coded according to survival probabilities: red (high-risk of mortality), green (intermediate-risk of mortality) and black (low-risk of mortality). Values in parentheses in the legend represent the number of activated risk pathways associated with each subgroup.

Figure 3. Comparison of the predictive performances between the pathway-level analysis (gcInGRiD) and the gene-level analysis (gene index count model) using the TCGA data. The predictive performances were compared based on the time-dependent area under the curve (AUC) at 60 months (left), the $-\log 10$ transformed $p$-value from global $F$-test (middle), and the subgroup distance (right). Subgroup distance is defined as the distance in the restricted mean event times between the high and low risk groups.

Figure 4. Kaplan-Meier curves of patient subgroups predicted by the pathway-level analysis (gcInGRiD) and the gene-level analysis (gene index count model) using the AOCS high-grade serous ovarian cancer data. Patient subgroups are color-coded according to survival probabilities: red (high-risk of mortality), green (intermediate-risk of 
mortality) and black (low-risk of mortality). Values in parentheses in the legend represent the number of pathways associated with each subgroup.

Figure 5. Comparison of the stability of the pathway-level analysis (gcInGRiD) and the gene-level analysis (gene index count model) in identifying molecular features and patient subgroups. 
1. Pre-filtering

Full set of genes

Univariate Cox regression

2. Gene-level analysis

Gene selection for pathway 1

3. Pathway-level analysis

\section{Patient subgrouping}

\section{Refined set of genes}

Genes in pathway 1

... Genes in pathway m

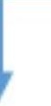

SPLS Cox regression

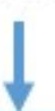

Latent components

for pathway 1

SPLS Cox regression

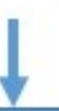

Latent components for pathway m
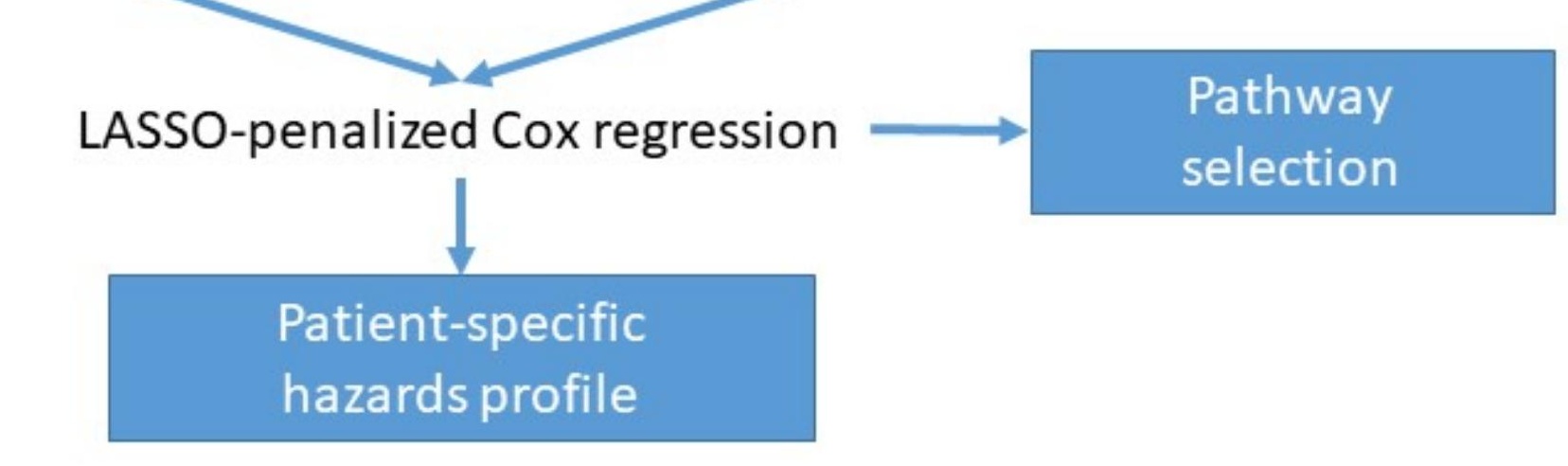
gclnGRiD

TCGA Data

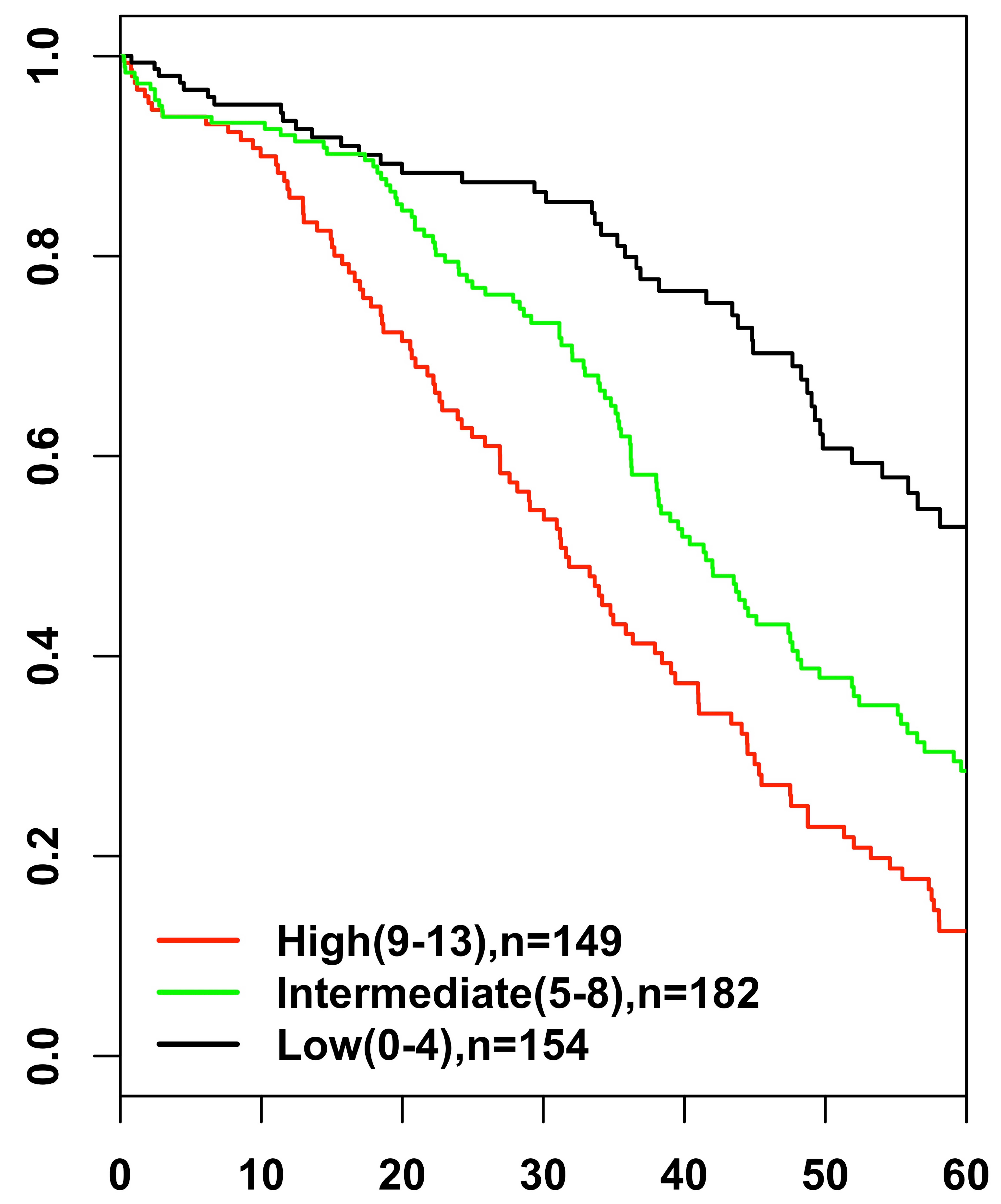

Months From Diagnosis to Death
Gene Index Count

TCGA Data

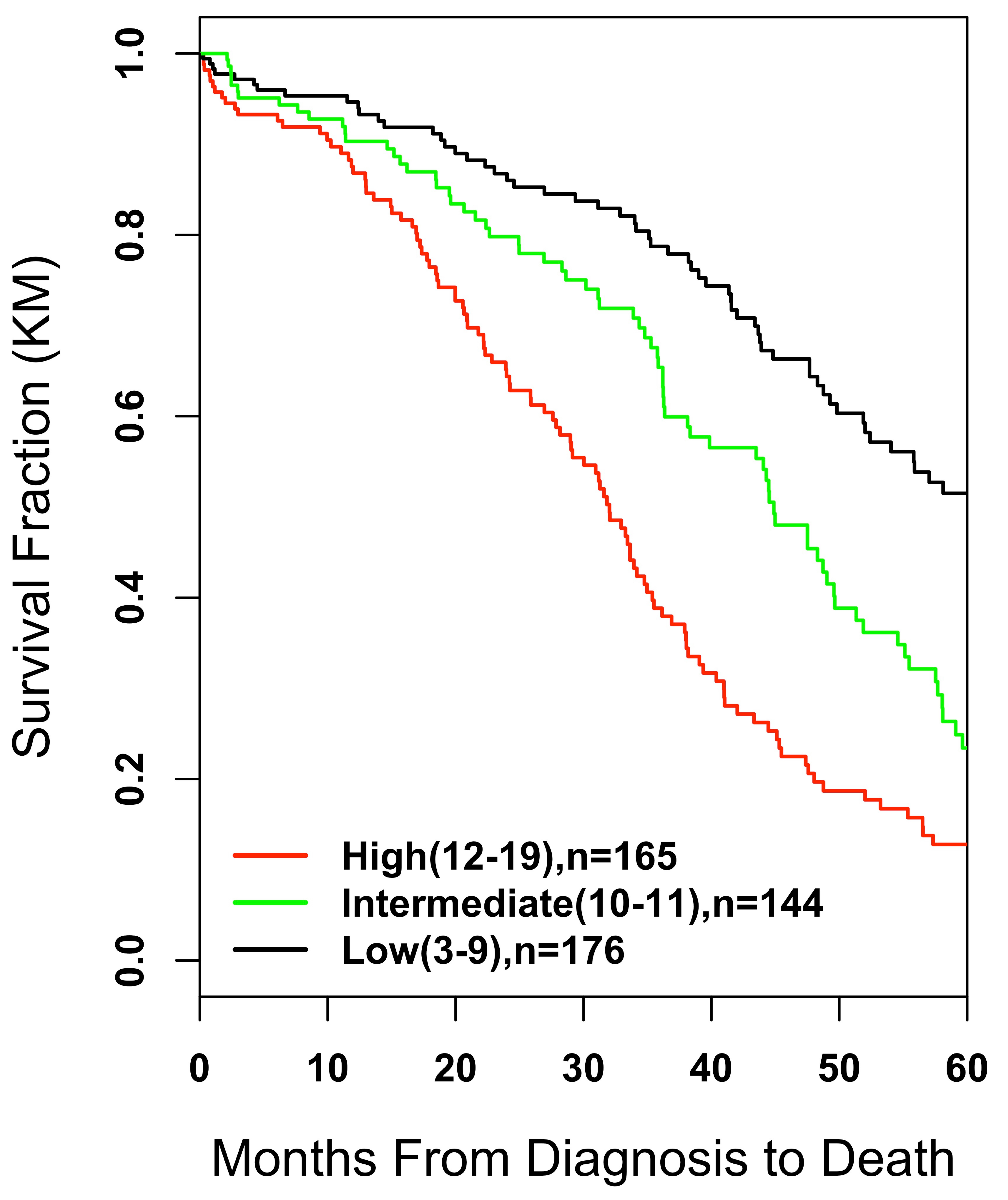




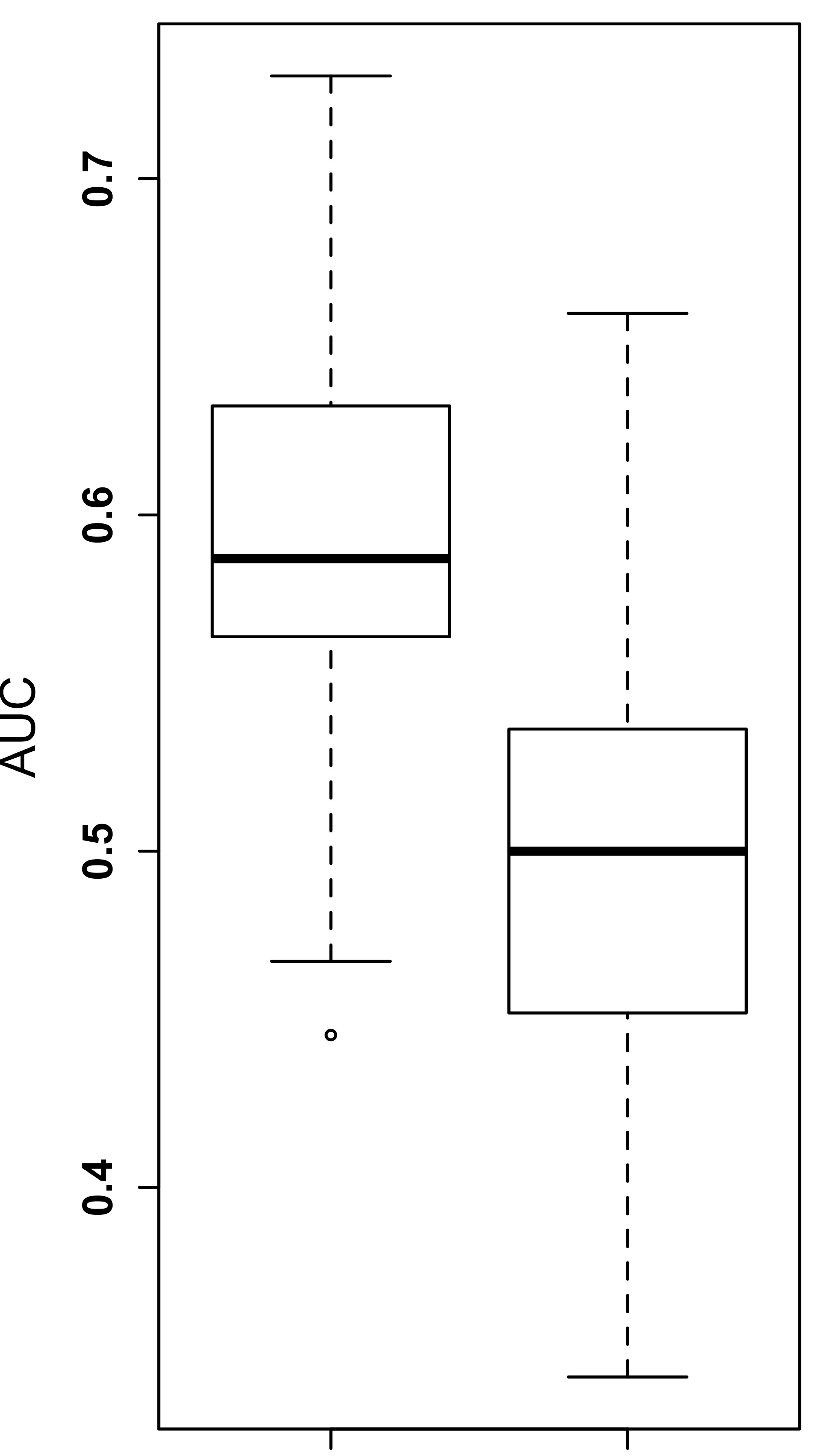

Pathway-level Gene-level

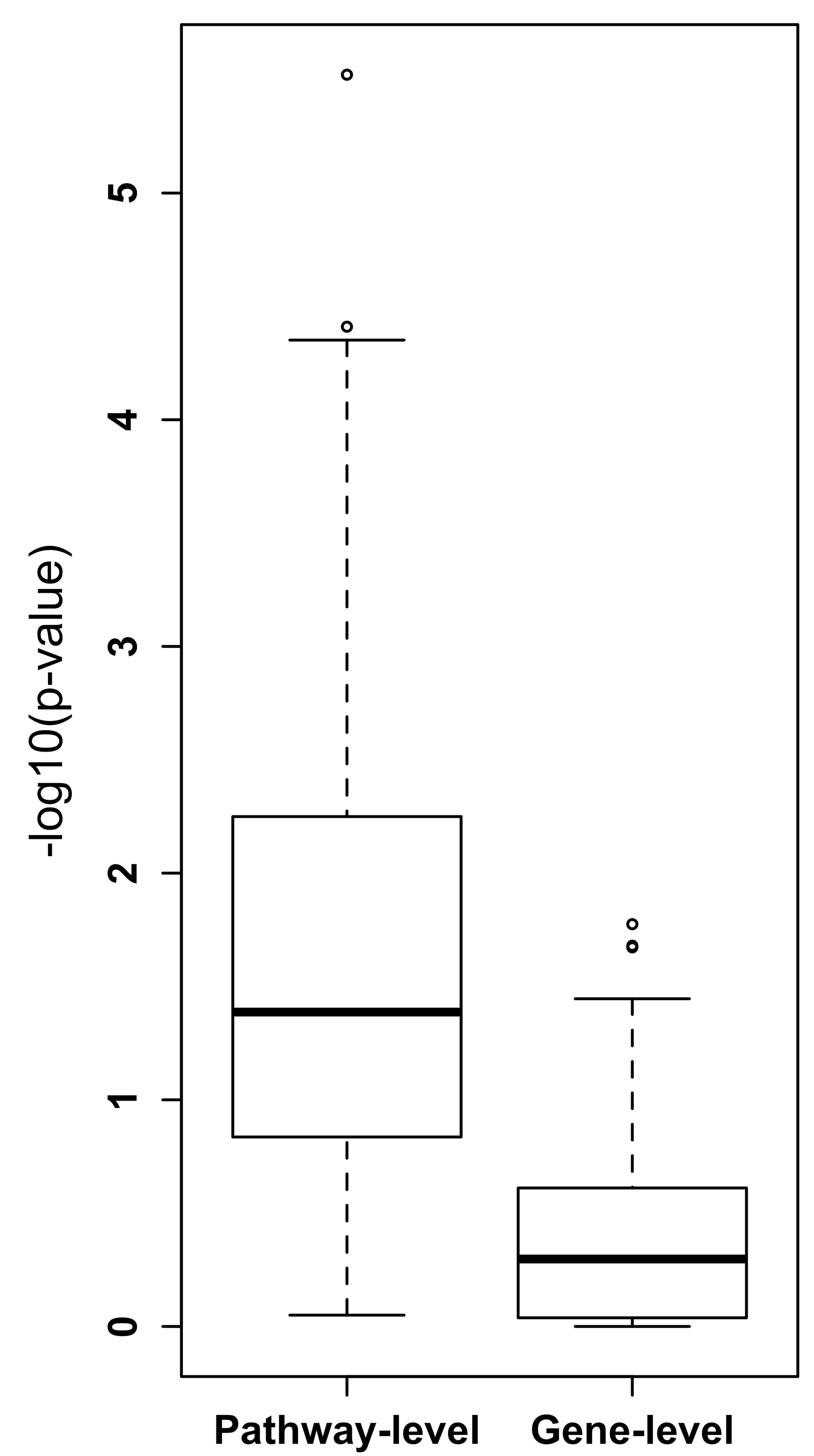

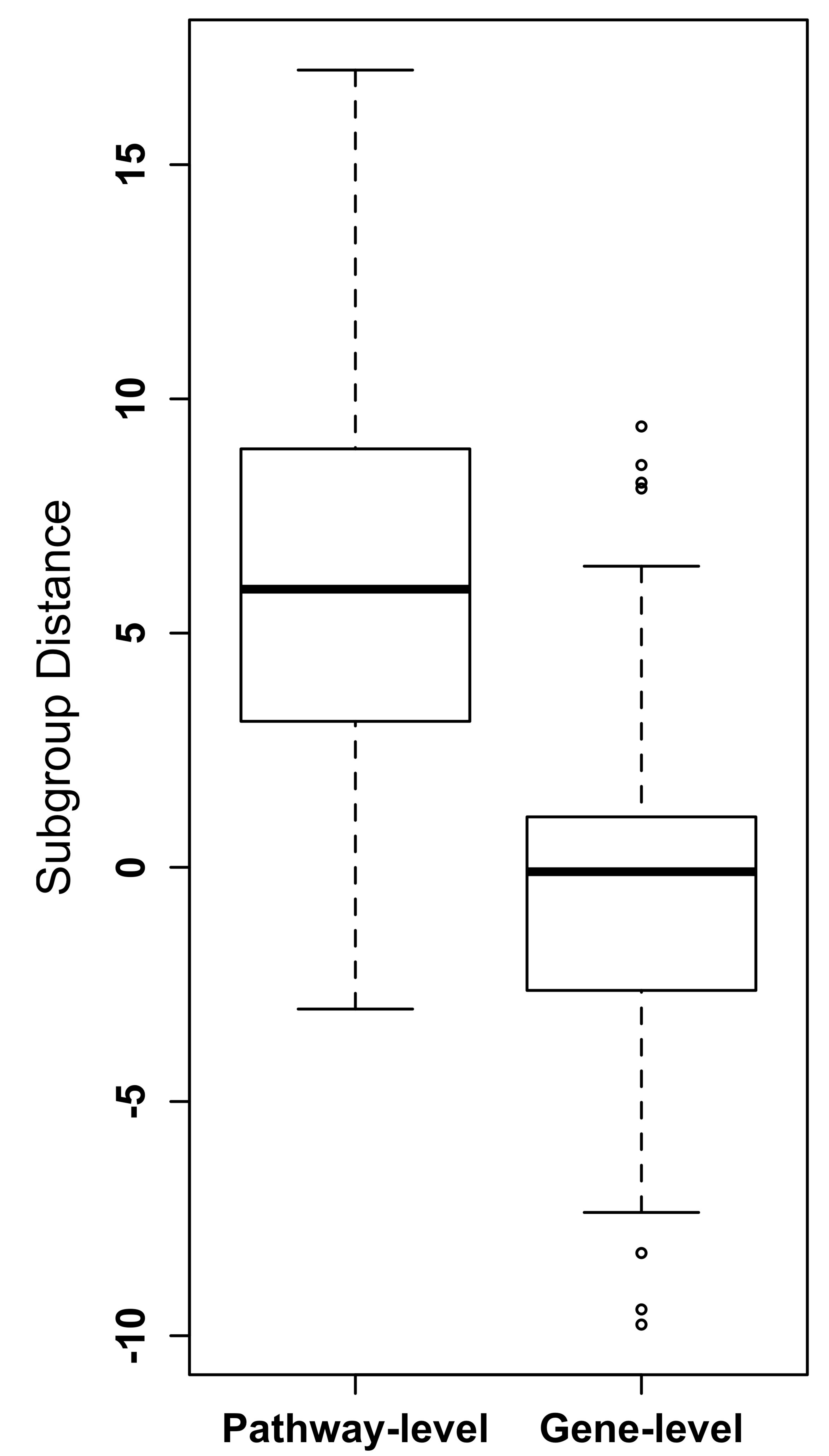


gclnGRiD

AOCS Data

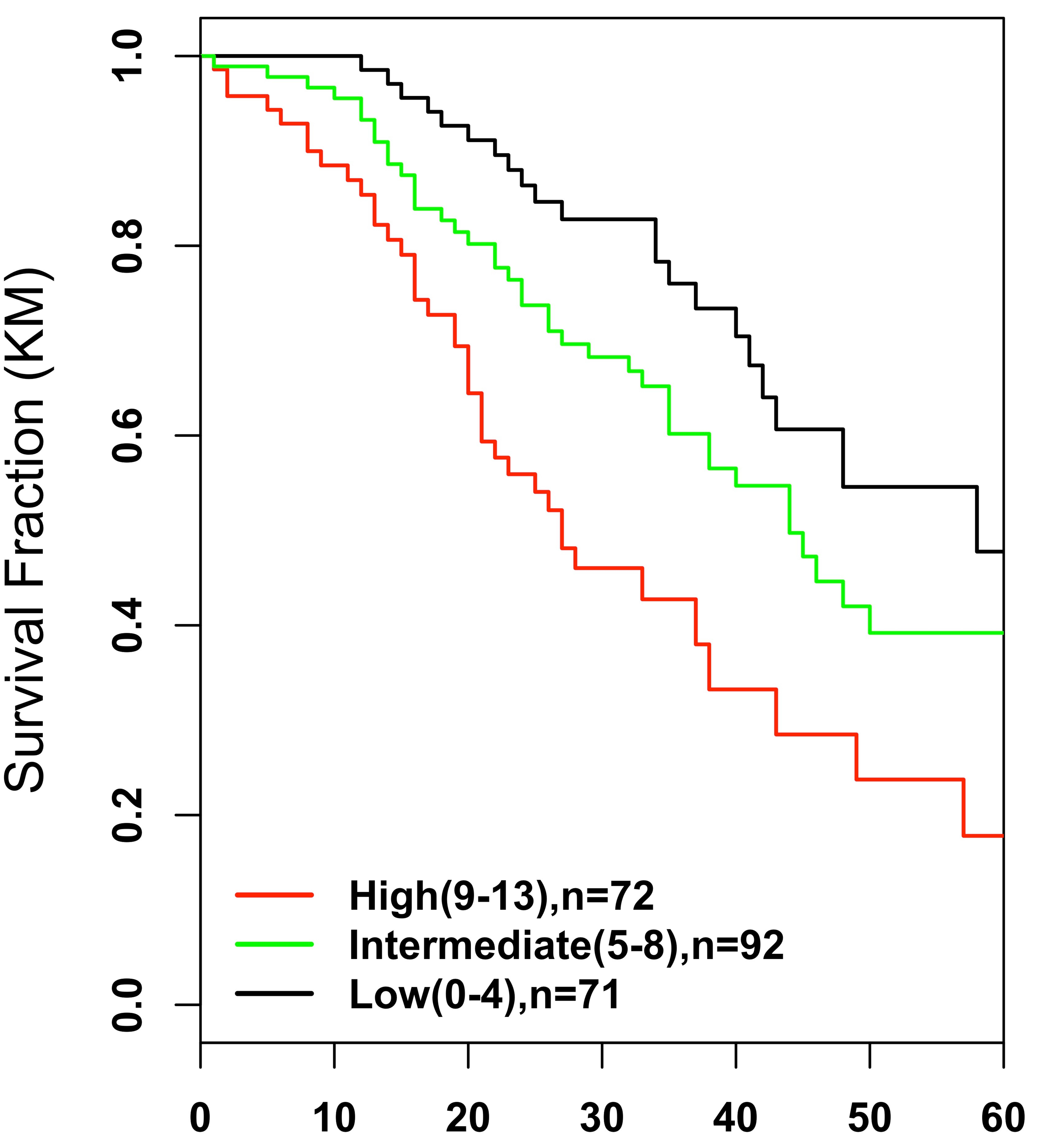

Months From Diagnosis to Death
Gene Index Count

AOCS Data

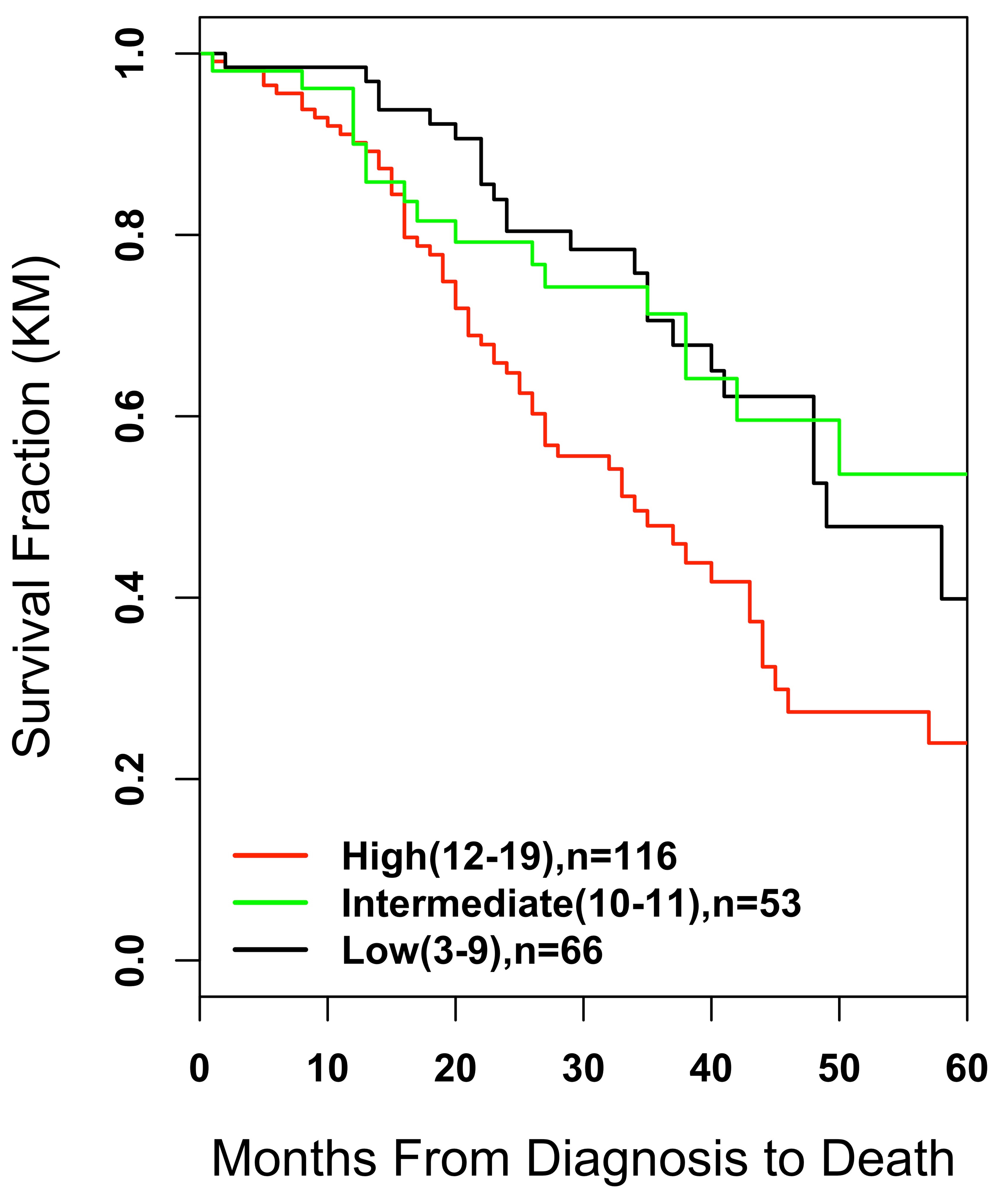


Supplemental Table 1: Top gene sets and genes selected by InGRiD based on the gene-cluster approach. Genes with $p$-values greater than $\mathbf{0 . 3 0}$ in the prefiltering step are not included in further analysis. The number of latent variables summarizing each gene set $\left(\boldsymbol{K}_{\boldsymbol{r}}\right)$ is fixed at one. Gene sets consisting of common genes across multiple pathways are written in bold face. The number within parenthesis in the column 'Genes Selected' refers to the total number of genes in each gene set.

\begin{tabular}{|c|c|c|c|c|c|}
\hline Gene Sets Selected & Genes & Gene Set & \multicolumn{3}{|c|}{ Top Three Genes } \\
\hline HEDGEHOG_SIGNALING & $8(20)$ & 0.207 & CSNK1G3 & GAS1 & CSNK1D \\
\hline MAPK\&APOPTOSIS & $26(68)$ & 0.113 & PРP3CA & RPS6KA2 & TGFBR1 \\
\hline MISMATCH_REPAIR & $3(7)$ & 0.083 & $S S B P 1$ & MLH3 & EXO1 \\
\hline PHOSPHATIDYLINOSITOL_SIGNALING_SYSTEM & $19(58)$ & 0.071 & PLCG1 & $P I P 4 K 2 B$ & IMPA2 \\
\hline NUCLEOTIDE_EXCISION_REPAIR & $6(19)$ & 0.067 & GTF2H4 & $D D B 2$ & ERCC1 \\
\hline NOTCH_SIGNALING & $1(36)$ & 0.057 & NOTCH4 & & \\
\hline CELL_ADHESION_MOLECULES_CAMS & $46(103)$ & 0.053 & CD6 & CTLA4 & CLDN6 \\
\hline JAK_STAT_SIGNALING & $44(115)$ & 0.044 & SOCS5 & $I L 21 R$ & IFNA21 \\
\hline BASE_EXCISION_REPAIR & $7(20)$ & 0.028 & MUTYH & $U N G$ & APEX1 \\
\hline MAPK_SIGNALING & $69(189)$ & 0.015 & $P L A 2 G 2 D$ & PDGFRA & $F G F 7$ \\
\hline WNT_SIGNALING & $30(67)$ & 0.010 & $A P C$ & CSNK2A2 & FZD1 \\
\hline DNA-REPAIR & $0(22)$ & 0 & & & \\
\hline WNT\&HEDGEHOG & $0(38)$ & 0 & & & \\
\hline TGF_BETA\&CELL_CYCLE\&WNT & $0(34)$ & 0 & & & \\
\hline NON_HOMOLOGOUS_END_JOINING & $0(10)$ & 0 & & & \\
\hline CELL_CYCLE & $0(84)$ & 0 & & & \\
\hline MTOR_SIGNALING & $0(29)$ & 0 & & & \\
\hline APOPTOSIS & $0(39)$ & 0 & & & \\
\hline TGF_BETA_SIGNALING & $0(43)$ & 0 & & & \\
\hline
\end{tabular}


Supplemental Table 2: Top gene sets and genes selected by InGRiD based on the gene-cluster approach. Genes with $p$-values greater than $\mathbf{0 . 7 0}$ in the prefiltering step are not included in further analysis. The number of latent variables summarizing each gene set $\left(\boldsymbol{K}_{r}\right)$ is fixed at one. Gene sets consisting of common genes across multiple pathways are written in bold face. The number within parenthesis in the column 'Genes Selected' refers to the total number of genes in each gene set.

\begin{tabular}{|c|c|c|c|c|c|}
\hline \multirow{2}{*}{$\begin{array}{l}\text { Gene Sets Selected } \\
\text { MAPK\&APOPTOSIS }\end{array}$} & \multirow{2}{*}{$\begin{array}{c}\text { Genes } \\
\text { Selected }\end{array}$} & \multirow{2}{*}{$\begin{array}{c}\begin{array}{c}\text { Gene Set } \\
\text { Coefficient }\end{array} \\
0.174\end{array}$} & \multicolumn{3}{|c|}{ Top Three Genes } \\
\hline & & & РРPЗСА & RPS6KA2 & TGFBRI \\
\hline MAPK_SIGNALING & $1(189)$ & 0.111 & PLA2G2D & & \\
\hline NOTCH_SIGNALING & 1(36) & 0.107 & NOTCH4 & & \\
\hline HEDGEHOG_SIGNALING & $2(20)$ & 0.106 & CSNK1G3 & GAS1 & \\
\hline CELL_CYCLE & 2(84) & 0.079 & MCM3 & ANAPC11 & \\
\hline PHOSPHATIDYLINOSITOL_SIGNALING_SYSTEM & $1(58)$ & 0.078 & PLCG1 & & \\
\hline NUCLEOTIDE_EXCISION_REPAIR & 2(19) & 0.076 & GTF2H4 & $D D B 2$ & \\
\hline MISMATCH_REPAIR & 1(7) & 0.059 & $S S B P 1$ & & \\
\hline CELL_ADHESION_MOLECULES_CAMS & $2(103)$ & 0.047 & CD6 & CTLA4 & \\
\hline WNT_SIGNALING & $44(67)$ & 0.021 & $A P C$ & CSNK2A2 & $F Z D 1$ \\
\hline WNT\&HEDGEHOG & $24(38)$ & 0.019 & $B T R C$ & $B M P 4$ & $W N T 8 B$ \\
\hline BASE_EXCISION_REPAIR & $8(20)$ & 0.013 & MUTYH & $U N G$ & APEXI \\
\hline DNA-REPAIR & $3(22)$ & 0.009 & POLD2 & FEN1 & POLD3 \\
\hline TGF_BETA\&CELL_CYCLE\&WNT & $0(34)$ & 0 & & & \\
\hline NON_HOMOLOGOUS_END_JOINING & $0(10)$ & 0 & & & \\
\hline MTOR_SIGNALING & $0(29)$ & 0 & & & \\
\hline TGF_BETA_SIGNALING & $0(43)$ & 0 & & & \\
\hline JAK_STAT_SIGNALING & $0(115)$ & 0 & & & \\
\hline APOPTOSIS & 2(39) & -0.034 & $A P A F 1$ & $\operatorname{IRAK2}$ & \\
\hline
\end{tabular}


Supplemental Table 3: Top gene sets and genes selected by InGRiD based on the gene-cluster approach. The number of latent variables summarizing each gene set $\left(K_{r}\right)$ is not fixed at one. Genes with $p$-values greater than $\mathbf{0 . 5 0}$ in the prefiltering step are not included in further analysis. In the table, the column "Gene Set Coefficients" means the $K_{r}$ coefficient estimates for the gene set $r$, where the optimal $K_{r}$ is determined based on the 10 -fold cross validation. Gene sets are ranked based on the number of genes selected from each gene set. Gene sets consisting of common genes across multiple pathways are written in bold face. The number within parenthesis in the column 'Genes Selected' refers to the total number of genes in each gene set.

\begin{tabular}{l|c|c|ccc}
\hline \multicolumn{1}{c|}{ Gene Sets Selected } & $\begin{array}{c}\text { Genes } \\
\text { Selected }\end{array}$ & $\begin{array}{c}\text { Gene Set } \\
\text { Coefficients }\end{array}$ & \multicolumn{3}{|c}{ Top Three Genes } \\
\hline MAPK_SIGNALING & $94(189)$ & 0.004 & PLA2G2D & PDGFRA & $F G F 7$ \\
JAK_STAT_SIGNALING & $67(115)$ & 0.058 & SOCS5 & $I L 21 R$ & $I F N A 21$ \\
CELL_ADHESION_MOLECULES_CAMS & $59(103)$ & 0.105 & CD6 & CTLA4 & CLDN6 \\
WNT_SIGNALING & $39(67)$ & 0.020 & APC & CSNK2A2 & $F Z D 1$ \\
MAPK\&APOPTOSIS & $37(68)$ & 0.062 & PPP3CA & RPS6KA2 & TGFBRI \\
PHOSPHATIDYLINOSITOL_SIGNALING & $30(58)$ & 0.015 & PLCG1 & PIP4K2B & IMPA2 \\
NUCLEOTIDE_EXCISION_REPAIR & $14(19)$ & $0.079,0.074$ & GTF2H4 & DDB2 & GTF2H5 \\
BASE_EXCISION_REPAIR & $10(20)$ & 0.015 & MUTYH & UNG & APEXI \\
HEDGEHOG_SIGNALING & $10(20)$ & $0.199,0.161$ & CSNK1D & ZIC2 & GASI \\
DNA-REPAIR & $3(22)$ & 0.017 & POLD2 & FEN1 & POLD3 \\
TGF_BETA\&CELL_CYCLE\&WNT & $3(34)$ & $0,0.107$ & PPP2RIB & IFNG & RPS6KB2 \\
MISMATCH_REPAIR & $2(7)$ & $0.148,-0.047$ & MLH3 & SSBP1 & \\
CELL_CYCLE & $2(84)$ & 0.003 & MCM3 & ANAPC11 & \\
NOTCH_SIGNALING & $1(36)$ & 0.056 & NOTCH4 & & \\
NON_HOMOLOGOUS_END_JOINING & $0(10)$ & 0 & & & \\
TGF_BETA_SIGNALING & $0(43)$ & 0 & & & \\
APOPTOSIS & $0(39)$ & 0 & & & \\
MTOR_SIGNALING & $0(29)$ & 0 & & & \\
WNT\&HEDGEHOG & $0(38)$ & 0 & & & \\
\hline
\end{tabular}


Supplemental Figure 1: Kaplan-Meier curves for patient subgroups identified from the TCGA data by gcInGRiD when $\boldsymbol{K}_{\boldsymbol{r}}$ is fixed at one. Different $p$-value cutoff points are considered in the prefiltering step: p-value<0.3 (Left Panel) and p-value<0.7 (Right Panel). Genes with pvalues greater than the cutoff points in the prefiltering step are not included in further analysis. Patient subgroups are color-coded according to survival probabilities: red (high-risk of mortality), green (intermediate-risk of mortality) and black (low-risk of mortality). Values in parentheses in the legend represent the number of activated risk pathways associated with each subgroup.
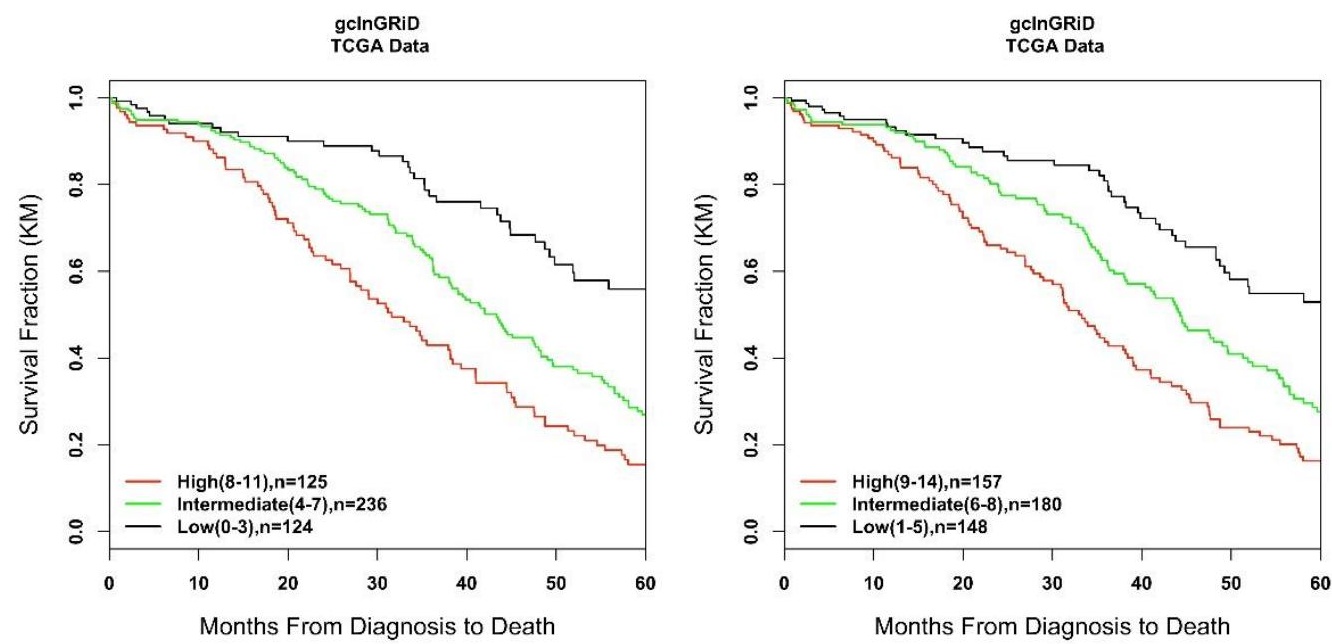
Supplemental Figure 2: Kaplan-Meier curves for patient subgroups identified from the TCGA data (training data) and the AOCS data (external validation) by gcInGRiD when $\boldsymbol{K}_{\boldsymbol{r}}$ is not fixed as one. Genes with p-values greater than 0.5 in the prefiltering step are not included in further analysis. The optimal number of latent variables summarizing each gene set $\left(K_{r}\right)$ is determined based on the 10-fold cross validation. Patient subgroups are color-coded according to survival probabilities: red (high-risk of mortality), green (intermediate-risk of mortality) and black (lowrisk of mortality). Values in parentheses in the legend represent the number of activated risk pathways associated with each subgroup.
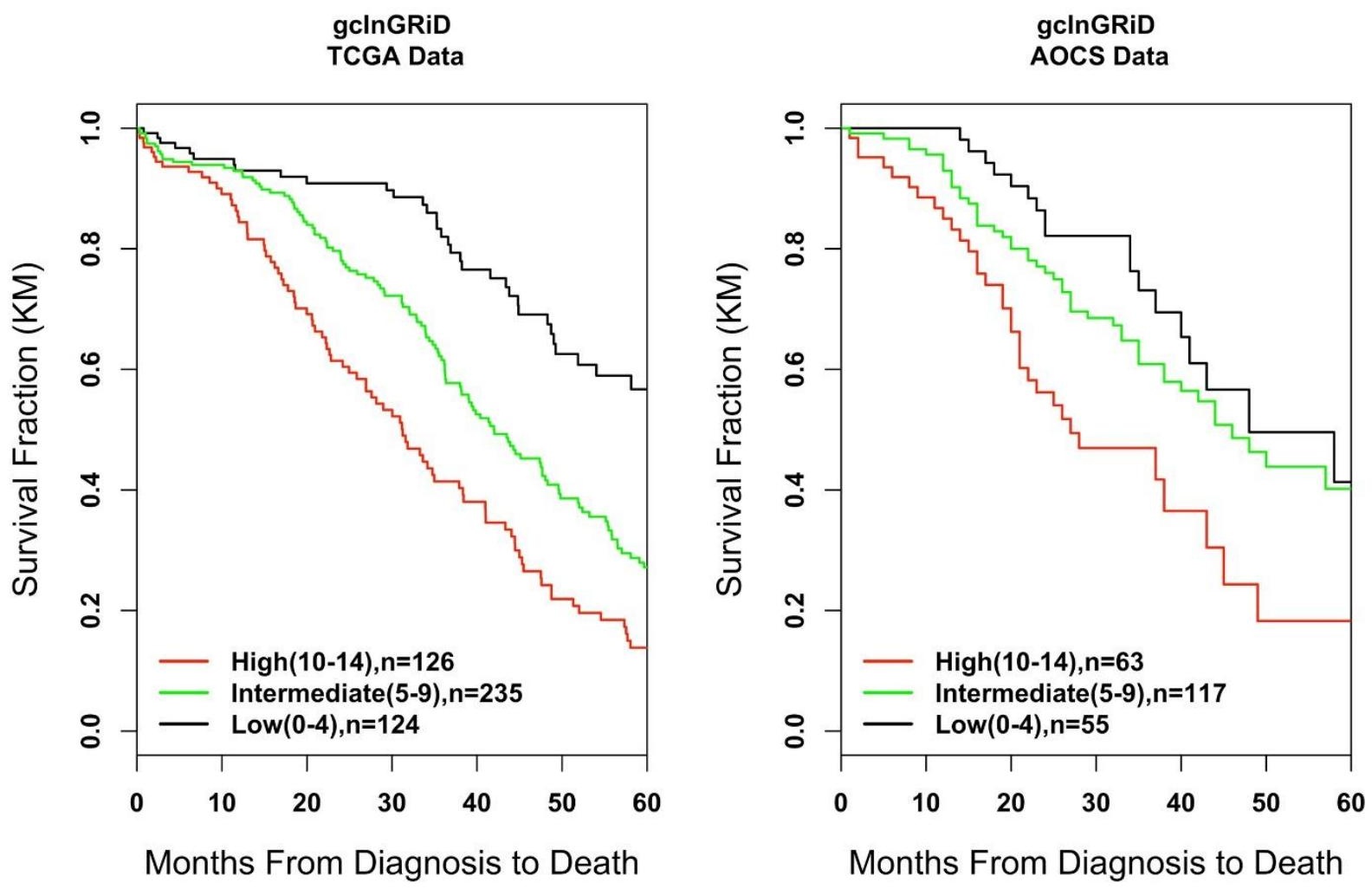
Supplemental Table 2. Novel gene sets identified by gclnGRiD from common genes shared across multiple pathways.

$\begin{array}{lcl}\text { DNA-REPAIR } & \text { WNT\&HEDGEHOG } \\ \text { POLE3 } & 1 & \text { PLCB2 } \\ \text { POLD3 } & 2 & \text { PLCB1 } \\ \text { POLD4 } & 3 & \text { PLCB3 } \\ \text { POLE2 } & 4 & \text { PLCB4 } \\ \text { POLD1 } & 5 & \text { GSK3B } \\ \text { POLD2 } & 6 & \text { BMP2 } \\ \text { POLE } & 7 & \text { BMP7 } \\ \text { POLE4 } & 8 & \text { BTRC } \\ \text { RFC4 } & 9 & \text { FBXW11 } \\ \text { RFC5 } & 10 & \text { CTBP2 } \\ \text { RPA1 } & 11 & \text { CTBP1 } \\ \text { RPA3 } & 12 & \text { WNT9B } \\ \text { RPA2 } & 13 & \text { WNT9A } \\ \text { PCNA } & 14 & \text { WNT2B } \\ \text { RFC1 } & 15 & \text { WNT11 } \\ \text { RFC3 } & 16 & \text { WNT10B } \\ \text { RFC2 } & 17 & \text { CSNK1A1 } \\ \text { FEN1 } & 18 & \text { WNT5B } \\ \text { LIG1 } & 19 & \text { WNT16 } \\ \text { POLL } & 20 & \text { DVL3 } \\ \text { CDK7 } & 21 & \text { DVL2 } \\ \text { CCNH } & 22 & \text { DVL1 } \\ & 23 & \text { WNT10A } \\ & 24 & \text { WNT4 } \\ & 25 & \text { WNT1 } \\ & 26 & \text { WNT7A } \\ & 27 & \text { WNT7B } \\ & 28 & \text { PSEN1 } \\ 29 & \text { WNT8A } \\ & 30 & \text { WNT8B } \\ & & \\ & & \\ & & \end{array}$

$\begin{array}{cl}\text { MAPK\&APOPTOSIS } \\ 1 & \text { PIK3CA } \\ 2 & \text { PIK3CB } \\ 3 & \text { PIK3CD } \\ 4 & \text { PIK3CG } \\ 5 & \text { JUN } \\ 6 & \text { GADD45B } \\ 7 & \text { MAP3K7 } \\ 8 & \text { AKT1 } \\ 9 & \text { AKT2 } \\ 10 & \text { CHP2 } \\ 11 & \text { NFKB1 } \\ 12 & \text { NFATC4 } \\ 13 & \text { TRAF2 } \\ 14 & \text { TGFBR2 } \\ 15 & \text { TGFBR1 } \\ 16 & \text { NFATC2 } \\ 17 & \text { MAPK9 } \\ 18 & \text { MAPK10 } \\ 19 & \text { CASP3 } \\ 20 & \text { RPS6KA2 } \\ 21 & \text { GRB2 } \\ 22 & \text { RPS6KA1 } \\ 23 & \text { PPP3CB } \\ 24 & \text { PPP3CA } \\ 25 & \text { PPP3R2 } \\ 26 & \text { PPP3CC } \\ 27 & \text { PPP3R1 } \\ 28 & \text { BRAF } \\ 29 & \text { IKBKB } \\ 30 & \text { IL1A } \\ & \end{array}$

\section{TGF_BETA\&CELL_CYCLE\&WNT}

1 IFNG

2 RBX1

3 PRKDC

$4 \quad$ MYC

5 TGFB2

6 TGFB1

7 TGFB3

8 RPS6KB1

9 RPS6KB2

10 ROCK1

11 RHOA

12 ROCK2

13 CREBBP

14 RBL2

15 RBL1

16 SKP1

17 CCND1

18 CCND2

19 CCND3

20 TFDP1

21 CUL1

22 SMAD3

23 SMAD4

24 SMAD2

25 EP300

26 CDKN2B

27 E2F5

28 E2F4

29 HDAC1

30 HDAC2 


$\begin{array}{ll}31 & \text { WNT2 } \\ 32 & \text { WNT5A } \\ 33 & \text { WNT6 } \\ 34 & \text { BMP8A } \\ 35 & \text { BMP4 } \\ 36 & \text { BMP8B } \\ 37 & \text { BMP5 } \\ 38 & \text { BMP6 }\end{array}$

$31 \quad$ AKT

32

33

34

35

36

37

38

39

40

41

42

43

44

45

46

47

48

49

50

51

52

53

54

55

59

60

61

62

63
AKT3

RELA

GADD45A

NGF

MAP3K14

PRKACA

PRKACB

PRKACG

CDC25B

SOS2

CHUK

PRKCB

PRKCA

TNF

PRKCG

RAC2

RAC3

TNFRSF1A

MAPK3

RAC1

SOS1

MAPK1

MAPK8

IL1B

IL1R1

TP53

FAS

FASLG

NTRK1

PIK3R5

PIK3R3

PIK3R1
31 PPP2CB

32 PPP2CA

33 PPP2R1A

34 PPP2R1B 
$64 \quad$ PIK3R2

65 CSF2RB

66 IL3

67 ATM

68 BCL2L1 